\title{
Uniqueness properties of degenerate elliptic operators
}

\author{
El MaAti Ouhabaz and DereK W. Robinson
}

Abstract. Let $\Omega$ be an open subset of $\mathbf{R}^{d}$ and $K=-\sum_{i, j=1}^{d} \partial_{i} c_{i j} \partial_{j}+\sum_{i=1}^{d} c_{i} \partial_{i}+c_{0}$ a second-order partial differential operator with real-valued coefficients $c_{i j}=c_{j i} \in W_{\mathrm{loc}}^{1, \infty}(\Omega), c_{i}, c_{0} \in L_{\infty, \operatorname{loc}}(\Omega)$ satisfying the strict ellipticity condition $C=\left(c_{i j}\right)>0$. Further let $H=-\sum_{i, j=1}^{d} \partial_{i} c_{i j} \partial_{j}$ denote the principal part of $K$. Assuming an accretivity condition $C \geq \kappa\left(c \otimes c^{T}\right)$ with $\kappa>0$, an invariance condition $\left(\mathbb{1}_{\Omega}, K \varphi\right)=0$ and a growth condition which allows $\|C(x)\| \sim|x|^{2} \log |x|$ as $|x| \rightarrow \infty$ we prove that $K$ is $L_{1}$-unique if and only if $H$ is $L_{1}$-unique or Markov unique.

\section{Introduction}

In this article, we examine criteria for a degenerate elliptic operator

$$
K=-\sum_{i, j=1}^{d} \partial_{i} c_{i j} \partial_{j}+\sum_{i=1}^{d} c_{i} \partial_{i}+c_{0},
$$

acting on $C_{c}^{\infty}(\Omega)$, where $\Omega$ is an open subset of $\mathbf{R}^{d}$, to be $L_{1}$-unique, i.e., to have a unique extension on $L_{1}(\Omega)$ which generates a continuous semigroup or to be Markov unique, i.e., to have a unique extension on $L_{2}(\Omega)$ which generates a submarkovian semigroup. This analysis continues earlier work of one of us with Adam Sikora [23,24] which studied these uniqueness properties for the principal part

$$
H=-\sum_{i, j=1}^{d} \partial_{i} c_{i j} \partial_{j}
$$

of $K$. We assume throughout that the coefficients are real, $c_{i j}=c_{j i} \in W_{\text {loc }}^{1, \infty}(\Omega), c_{i}, c_{0}$ $\in L_{\infty, \text { loc }}(\Omega)$ and that the diffusion matrix $C=\left(c_{i j}\right)$ is strictly elliptic, i.e., $C(x)>0$ for all $x \in \Omega$. In addition, we allow a possible growth of the $c_{i j}$ at infinity. (For general background on uniqueness properties of diffusion operators, we rely on the lecture notes of Eberle [7] and the references cited therein.)

In general, one cannot expect the evolution process associated with $K$ to be unique if $\Omega$ has a boundary. The principal part $H$ describes a diffusion which is only uniquely 
defined if the boundary behavior is also specified. There are various possible phenomena modeled by boundary conditions, transmission, reflection, absorption, etc., each of which leads to a different global process. If, however, the matrix $C$ is sufficiently degenerate at the boundary then the corresponding diffusion does not reach the boundary and consequently is independent of boundary conditions. In particular, it was established in $[23,24]$ that $H$ is $L_{1}$-unique, or Markov unique, if and only if the semigroup $S$ generated by the Friedrichs extension of $H$ satisfies the invariance property $\left(\mathbb{1}_{\Omega}, S_{t} \varphi\right)=\left(\mathbb{1}_{\Omega}, \varphi\right)$ for all $\varphi \in L_{1}(\Omega)$ and all $t>0$. The addition, however, of lower-order terms complicates the situation. In this article, we concentrate on the complications introduced by the first-order terms $c . \nabla=\sum_{i=1}^{d} c_{i} \partial_{i}$.

The first-order terms $c$. $\nabla$ superimpose a flow, or drift, with velocity $c$ onto the diffusion process. The flow can then force the diffusion onto the boundary and it is again necessary to specify boundary conditions for a complete description of the evolution. The aim of this article is to show that the uniqueness properties established for $H$ in $[23,24]$ are preserved if the first-order terms satisfy two general restraints. First, we assume the degeneracy and growth properties of the drift coefficients are dictated by the properties of the leading order coefficients $c_{i j}$. In particular, we assume there is a $v>0$ such that

$$
C(x) \geq v\left(c \otimes c^{T}\right)(x)
$$

for all $x \in \Omega$. Thus, the norm $\|C(x)\|$ of the matrix of principal coefficients dominates the first-order coefficients, $\|C(x)\| \geq v|c(x)|^{2}$, and if the diffusion matrix $C$ is degenerate at the boundary in the sense that $\|C(x)\| \rightarrow 0$ as $x \rightarrow \partial \Omega$ then the velocity of the flow also tends to zero at the boundary. Secondly, we assume that $K$ satisfies the infinitesimal invariance property $\left(\mathbb{1}_{\Omega}, K \varphi\right)=0$ for all $\varphi \in C_{c}^{\infty}(\Omega)$. Since the principal part $H$ of $K$ is in divergence form this is equivalent to the condition $\left(\mathbb{1}_{\Omega},\left(c . \nabla+c_{0}\right) \varphi\right)=0$ for all $\varphi \in C_{c}^{\infty}(\Omega)$. Alternatively, this can be phrased as $c_{0}=\operatorname{div} c$ in the sense of distributions. In particular, this covers the case of an incompressible flow, $\operatorname{div} c=0$. In addition, we allow the coefficients of $H$ to grow at infinity. The growth restraint, which will be introduced in Sects. 2 and 3, allows $\|C(x)\| \sim|x|^{2} \log |x|$ as $|x| \rightarrow \infty$.

Our principal result states that the accretivity assumption (3) together with a slightly strengthened version of the invariance condition together with the growth restraint imply that $K$ is $L_{1}$-unique if and only if $H$ is $L_{1}$-unique or if and only if $S$ is $L_{1}$-invariant. Therefore, one can use the criteria of the earlier papers $[23,24]$ for $L_{1}$-uniqueness of the symmetric operator $H$ to deduce the corresponding uniqueness of the non-symmetric operator $K$. For example, $L_{1}$-uniqueness of $K$ is then equivalent to Markov uniqueness of $H$. Alternatively, $L_{1}$-uniqueness of $K$ is equivalent to the capacity $\operatorname{cap}(\partial \Omega)$ of the boundary $\partial \Omega$ of $\Omega$ measured with respect to $H$ being zero. These criteria will be discussed in Sect. 4 . In Sect. 2, we derive accretivity and continuity properties of various extensions of $K$ corresponding to different Dirichlet and Neumann boundary conditions. Then, in Sect. 3 , we establish two key invariance 
properties for semigroups generated by extensions of $K$ with these boundary conditions. Finally, we discuss some examples of one-dimensional differential operators that show the relevance of our assumptions.

\section{Accretivity, closability, and continuity}

In this section, we consider the definition of the degenerate elliptic operators $K$ and $H$ by the use of accretive forms. In general, we adopt the definitions and conventions of [21]. As a precursor to the discussion of the non-symmetric operator $K$, we first define precisely the principal part $H$.

Consider the symmetric bilinear form $h$ defined on $L_{2}(\Omega)$ by

$$
h(\varphi, \psi)=\sum_{i, j=1}^{d}\left(\partial_{i} \varphi, c_{i j} \partial_{j} \psi\right)
$$

for all (real-valued) $\varphi, \psi \in C_{c}^{\infty}(\Omega)$ and let $h(\varphi)=h(\varphi, \varphi)$ denote the corresponding quadratic form. We assume that the coefficients are real, that $c_{i j}=c_{j i} \in W_{\mathrm{loc}}^{1, \infty}(\Omega)$ and the matrix $C=\left(c_{i j}\right)$ satisfies the strict ellipticity condition $C>0$. Then, $h$ is the form of the positive symmetric operator $H$ with domain $D(H)=C_{c}^{\infty}(\Omega)$ defined by (2). It follows that $h$ is closable and its closure $\bar{h}$ determines a positive self-adjoint extension $H_{D}$ of $H$. The notation $H_{D}$ is chosen since this extension corresponds formally to the self-adjoint extension of the operator $H$ with Dirichlet boundary conditions on the boundary $\partial \Omega$ of $\Omega$. For the consistency of notation, we then set $\bar{h}=h_{D}$. The form $h_{D}$ is automatically a Dirichlet form. Therefore, the self-adjoint semigroup $T^{D}$ generated by (minus) $H_{D}$ is a submarkovian semigroup. In particular, $T^{D}$ is positive and $L_{\infty}$-contractive. We shall say that $H_{D}$ is a submarkovian extension of $H$.

Next, we introduce a second submarkovian extension of $H$ which can be interpreted as the extension corresponding to Neumann boundary conditions. Since we are not assuming any smoothness of the boundary $\partial \Omega$, the notion of Neumann boundary conditions is somewhat ambiguous but the definition we adopt is convenient for the analysis of uniqueness properties. Define the quadratic form $h_{N}$ by first introducing the domain

$$
D\left(h_{N}\right)=\left\{\varphi \in W_{\mathrm{loc}}^{1,2}(\Omega): \Gamma(\varphi)+\varphi^{2} \in L_{1}(\Omega)\right\}
$$

where $\Gamma$, the carré $d u$ champ of $H$, denotes the positive map

$$
\varphi \in W_{\mathrm{loc}}^{1,2}(\Omega) \mapsto \Gamma(\varphi)=\sum_{i, j=1}^{d} c_{i j}\left(\partial_{i} \varphi\right)\left(\partial_{j} \varphi\right) \in L_{1, \mathrm{loc}}(\Omega) .
$$

Then define $h_{N}$ by

$$
h_{N}(\varphi)=\int_{\Omega} \Gamma(\varphi)
$$

for all $\varphi \in D\left(h_{N}\right)$. The corresponding bilinear form is defined by polarization. 
PROPOSITION 2.1. The form $h_{N}$ is positive, densely defined, and closed. The corresponding positive self-adjoint operator $H_{N}$ is a submarkovian extension of the operator $H$ given by (2).

Proof. The form is clearly positive and densely defined. To prove that it is closed let $U$ be a relatively compact subset of $\Omega$. Then there exist $\mu_{U}, v_{U}>0$ such that $\mu_{U} I \leq C(x) \leq v_{U} I$ for all $x \in U$, i.e., $C$ is locally strongly elliptic. Therefore the form

$$
\varphi \in W^{1,2}(U) \mapsto \int_{U} \Gamma(\varphi)
$$

is closed. But

$$
h_{N}(\varphi)=\sup _{U \in \mathcal{D}} \int_{U} \Gamma(\varphi)=\lim _{U \in \mathcal{D}} \int_{U} \Gamma(\varphi)
$$

for all $\varphi \in D\left(h_{N}\right)$ where the supremum and limit are over the directed set $\mathcal{D}$ formed by the relatively compact subsets $U$ of $\Omega$ ordered by inclusion. Thus, $h_{N}$ is the supremum of a family of closed forms. Then, it follows by standard monotonicity arguments that $h_{N}$ is closed (see, for example, [16], Section VIII.3.4 or [17] Proposition I.3.9).

Next, a straightforward calculation establishes that

$$
h_{N}(\psi, \varphi)=(H \psi, \varphi)
$$

for all $\varphi \in D\left(h_{N}\right)$ and $\psi \in C_{c}^{\infty}(\Omega)$. Therefore, the self-adjoint operator $H_{N}$ associated with $h_{N}$ is an extension of $H$ (see [16], Corollary 2.4 in Section VI.2). But $h_{N}$ is a Dirichlet form by standard considerations. Hence, $H_{N}$ is a submarkovian extension of $H$.

In general, the two submarkovian extensions $H_{D}$ and $H_{N}$ of $H$ are distinct. But since $h_{N} \supseteq h_{D}$, it follows that one has the ordering $0 \leq H_{N} \leq H_{D}$. The foregoing definition of $h_{N}$ corresponds to the definitions given in [23,24] for the maximal Dirichlet form extension of $h$. The form $h_{D}$ is clearly the smallest Dirichlet form extension. Moreover, the semigroup $T^{N}$ generated by $H_{N}$ dominates $T^{D}$ in the sense that $T_{t}^{D} \varphi \leq T_{t}^{N} \varphi$ for all positive $\varphi$ and all $t>0$ (see, for example, [21] Theorem 2.24).

Since $h_{N}$ is a Dirichlet form, it follows that $D\left(h_{N}\right) \cap L_{\infty}(\Omega)$ is a core of $h_{N}$, i.e., the subspace is dense in $D\left(h_{N}\right)$ with respect to the norm $\varphi \mapsto\|\varphi\|_{D\left(h_{N}\right)}=$ $\left(h_{N}(\varphi)+\|\varphi\|_{2}^{2}\right)^{1 / 2}$. But if the coefficients $c_{i j}$ do not grow too fast then one has a stronger result.

Let $\|C(x)\|$ denote the norm of the matrix $C(x)=\left(c_{i j}(x)\right)$. Assume without loss of generality that $0 \in \Omega$ and introduce the strictly positive, non-decreasing, function $r \in\langle 0, \infty\rangle \mapsto v(r)>0$ by

$$
v(r)=\sup \{\|C(x)\|: x \in \Omega,|x|<r\} .
$$

Then $\|C(x)\| \leq v(|x|)$. Next define the positive increasing function $\mu$ by

$$
s \in\langle 0, \infty\rangle \mapsto \mu(s)=\int_{0}^{s} \mathrm{~d} t v(t)^{-1 / 2} .
$$


In the sequel, we often assume the following growth condition.

(Asymptotic growth I)

$$
\lim _{s \rightarrow \infty} \mu(s)=\infty
$$

If $\Omega$ is bounded and the coefficients $c_{i j}$ are bounded up to the boundary then $v$ is constant for $r \geq \operatorname{diam}(\Omega)$ and (8) is automatically satisfied. In general, however, (8) is an implicit restraint on the growth of the coefficients at the boundary or at infinity.

The function $\mu(|x|)$ is a lower bound on the Riemannian distance from 0 to $x$ measured with respect to the metric $C^{-1}$. So the growth condition ensures that the Riemannian distance to infinity is infinite.

REMARK 2.2. If $\|C(x)\|$ satisfies a quadratic growth bound, i.e., if there is an $a>0$ such that $v(r)=a\left(1+r^{2}\right)$ for all $x \in \Omega$, then $\mu(s) \sim \log s$ as $s \rightarrow \infty$ and (8) is satisfied. More generally, if $v(r) \sim r^{2}(\log r)^{\alpha}$ as $x \rightarrow \infty$ with $\alpha \geq 0$ then $\mu(s) \sim(\log s)^{1-\alpha / 2}$ as $s \rightarrow \infty$ and (8) is satisfied for $\alpha \in[0,2\rangle$.

Condition (8) has been used by various authors to establish $L_{2}$-properties of elliptic operators such as essential self-adjointness (see, for example, [4,7] and [24]). More stringent bounds are, however, often required to derive comparable $L_{1}$-properties (see Sect. 3).

LEMMA 2.3. Assume the growth condition (8) is satisfied. It follows that $\left(D\left(h_{N}\right) \cap\right.$ $\left.L_{\infty}(\Omega)\right)_{c}$, the bounded functions in $D\left(h_{N}\right)$ with compact support in $\bar{\Omega}$, is a core of $h_{N}$. Therefore, $D\left(h_{N}\right) \cap W^{1,2}(\Omega)$ is a core of $h_{N}$.

Proof. Let $\chi \in C_{c}^{\infty}(\mathbf{R})$ satisfy $0 \leq \chi \leq 1, \chi(s)=1$ if $s \in[0,1\rangle, \chi(x)=0$ if $s \geq 2$ and $\left|\chi^{\prime}\right| \leq 2$. Then define $\eta_{n}$ by $x \in \Omega \mapsto \eta_{n}(x)=\chi\left(n^{-1} \mu(|x|)\right)$. It follows from the growth condition (8) that the $\eta_{n}$ have compact support and $\eta_{n}(x) \rightarrow 1$ as $n \rightarrow \infty$ for all $x \in \Omega$. Moreover,

$$
0 \leq \Gamma\left(\eta_{n}\right) \leq \sup _{x \in \Omega} v(|x|)\left|\left(\nabla \eta_{n}\right)(x)\right|^{2} \leq 4 n^{-2} \sup _{x \in \Omega} v(|x|)|(\nabla \mu)(|x|)|^{2} \leq 4 n^{-2} .
$$

In particular $\Gamma\left(\eta_{n}\right)(x) \rightarrow 0$ as $n \rightarrow \infty$ for all $x \in \Omega$.

Next fix $\varphi \in D\left(h_{N}\right) \cap L_{\infty}(\Omega)$ and set $\varphi_{n}=\eta_{n} \varphi$. Then $\varphi_{n} \in\left(D\left(h_{N}\right) \cap L_{\infty}(\Omega)\right)_{c}$. But

$$
\left\|\varphi_{n}-\varphi\right\|_{2}^{2}=\left\|\left(\mathbb{1}_{\Omega}-\eta_{n}\right) \varphi\right\|_{2}^{2}=\int_{\Omega}\left(\mathbb{1}_{\Omega}-\eta_{n}\right)^{2} \varphi^{2}
$$

converges to zero as $n \rightarrow \infty$ by the dominated convergence theorem. In addition

$$
h_{N}\left(\varphi_{n}-\varphi\right)=h_{N}\left(\left(\mathbb{1}_{\Omega}-\eta_{n}\right) \varphi\right) \leq 2 \int_{\Omega} \Gamma\left(\eta_{n}\right) \varphi^{2}+2 \int_{\Omega}\left(\mathbb{1}_{\Omega}-\eta_{n}\right)^{2} \Gamma(\varphi) .
$$

But both terms on the right converge to zero as $n \rightarrow \infty$ by dominated convergence. The first converges to zero since $\varphi \in L_{2}(\Omega)$ and $\Gamma\left(\eta_{n}\right) \rightarrow 0$ and the second since $\varphi \in D\left(h_{N}\right)$ and $\eta_{n} \rightarrow \mathbb{1}_{\Omega}$. Therefore, $\varphi \in D\left(h_{N}\right) \cap L_{\infty}(\Omega)$ is the limit with respect 
to the $D\left(h_{N}\right)$-graph norm of the $\varphi_{n} \in\left(D\left(h_{N}\right) \cap L_{\infty}(\Omega)\right)_{c}$. Since $D\left(h_{N}\right) \cap L_{\infty}(\Omega)$ is a core of $h_{N}$, it follows that $\left(D\left(h_{N}\right) \cap L_{\infty}(\Omega)\right)_{c}$ is also a core.

The final statement of the lemma follows from the foregoing argument because $D\left(h_{N}\right) \subseteq W_{\mathrm{loc}}^{1,2}(\Omega)$. Therefore, if $\varphi \in D\left(h_{N}\right)$ then $\varphi_{n}=\eta_{n} \varphi \in D\left(h_{N}\right) \cap W^{1,2}(\Omega)$.

Next, we consider a different approximation procedure for constructing extensions of $H$.

Let $h_{D}^{(n)}$ and $h_{N}^{(n)}, n \in \mathbf{N}$, denote the forms with Dirichlet and Neumann boundary conditions associated with the elliptic operator $H^{(n)}$ defined by (2) but with the coefficients $c_{i j}$ replaced by $c_{i j}^{(n)}=c_{i j}+n^{-1} \delta_{i j}$. Further let $H_{D}^{(n)}, H_{N}^{(n)}$ denote the corresponding extensions of $H^{(n)}$. Since $C^{(n)}>n^{-1} I$ the $H^{(n)}$ are strongly elliptic operators. Moreover, if $l_{D}$ and $l_{N}$ are the forms associated with the Dirichlet and Neumann Laplacians on $\Omega$, i.e., if $D\left(l_{N}\right)=W^{1,2}(\Omega)$ and $l_{N}(\varphi)=\|\nabla \varphi\|_{2}^{2}$ and if $l_{D}$ is the restriction of $l_{N}$ to $W_{0}^{1,2}(\Omega)$, then $h_{D}^{(n)}=h_{D}+n^{-1} l_{D}$ with $D\left(h_{D}^{(n)}\right)=D\left(h_{D}\right) \cap W_{0}^{1,2}(\Omega)$ and $h_{N}^{(n)}=h_{N}+n^{-1} l_{N}$ with $D\left(h_{N}^{(n)}\right)=D\left(h_{N}\right) \cap W^{1,2}(\Omega)$.

PROPOSITION 2.4. Assume the coefficients of $H$ satisfy the growth restraint (8). The operators $H_{D}^{(n)}$ converge in the strong resolvent sense to $H_{D}$ as $n \rightarrow \infty$. Similarly, the operators $H_{N}^{(n)}$ converge in the strong resolvent sense to $H_{N}$.

Proof. The sequence of symmetric forms $h_{D}^{(n)}$ is decreasing and $h_{D}^{(n)} \geq h_{D}$. Therefore, the $H_{D}^{(n)}$ converge in the strong resolvent sense to a positive self-adjoint operator $\widehat{H}$ by [16], Theorem VIII.3.13. Moreover, $h_{D}(\varphi)=\lim _{n \rightarrow \infty} h_{D}^{(n)}(\varphi)$ for all $\varphi \in D\left(h_{D}\right) \cap W_{0}^{1,2}(\Omega)=\bigcap_{n \geq 1} D\left(h_{D}^{(n)}\right)$. Therefore, the form $\hat{h}$ of $\widehat{H}$ is the closure of $\left.h_{D}\right|_{D\left(h_{D}\right) \cap W_{0}^{1,2}(\Omega)}$ again by [16], Theorem VIII.3.13. But $C_{c}^{\infty}(\Omega) \subseteq D\left(h_{D}\right) \cap W_{0}^{1,2}(\Omega)$ and $C_{c}^{\infty}(\Omega)$ is a core of $h_{D}$. Therefore $\hat{h}=h_{D}$ and $\widehat{H}=H_{D}$.

It follows from another application of Kato's theorem that the operators $H_{N}^{(n)}$ converge in the strong resolvent sense to the operator associated with the closure of $\left.h_{N}\right|_{D\left(h_{N}\right) \cap W^{1,2}(\Omega)}$. But $D\left(h_{N}\right) \cap W^{1,2}(\Omega)$ is a core of $h_{N}$ by Lemma 2.3. This is the point that uses the growth condition (8). Therefore, the $H_{N}^{(n)}$ converge to $H_{N}$.

The strong resolvent convergence on $L_{2}(\Omega)$ of the strongly elliptic operators $H_{N}^{(n)}$ to the submarkovian extension $H_{N}$ of $H$ established by Proposition 2.4 is equivalent to the strong $L_{2}$-convergence of the submarkovian semigroups $S^{N,(n)}$ generated by the $H_{N}^{(n)}$ to the semigroup $S^{N}$ generated by $H_{N}$. This is the basis of the subsequent discussion of conservation and uniqueness properties (see Sect. 3).

Next, we consider the addition of first-order and zero-order terms to the operator $H$, i.e., we now analyze the operator $K$ defined by (1). We assume that the degeneracy and growth properties of the first-order coefficients $c=\left(c_{1}, \ldots, c_{d}\right)$ are dictated by the properties of the leading order coefficients $c_{i j}$. In particular, we assume there is a $v>0$ such that the matrix condition

(Uniform accretivity)

$$
C(x) \geq v\left(c \otimes c^{T}\right)(x),
$$


is satisfied for all $x \in \Omega$. Then we set $\kappa=\sup v$, i.e., $\kappa$ is the largest value for which (9) is valid. This accretivity assumption immediately implies that

$$
|c . \nabla \varphi|^{2} \leq \kappa^{-1} \Gamma(\varphi)
$$

for all $\varphi \in W_{\text {loc }}^{1,2}(\Omega)$. Hence $c . \nabla$ extends to an operator on $D\left(h_{N}\right)$ with

$$
\|c . \nabla \varphi\|_{2}^{2} \leq \kappa^{-1} h_{N}(\varphi)
$$

for all $\varphi \in D\left(h_{N}\right)$. Therefore

$$
\|c . \nabla \varphi\|_{2}^{2} \leq \varepsilon\left\|H_{N} \varphi\right\|_{2}^{2}+(4 \varepsilon \kappa)^{-1}\|\varphi\|_{2}^{2}
$$

for all $\varepsilon>0$ and all $\varphi \in D\left(H_{N}\right)$. Hence, one can use perturbation theory to deduce that $H_{N}+c$. $\nabla$ generates an $L_{2}$-continuous semigroup. Restricting (10) to $\varphi \in D\left(h_{D}\right)$ one deduces similarly that $H_{D}+c . \nabla$ is a generator. Since we intend to analyze conservation properties, it is, however, necessary to introduce an additional invariance assumption involving the zero-order term and to proceed slightly differently in defining the corresponding generators.

We assume that $K$ satisfies the invariance condition $\left(\mathbb{1}_{\Omega}, K \varphi\right)=0$ for all $\varphi \in$ $C_{c}^{\infty}(\Omega)$. But this is equivalent to $\left(\mathbb{1}_{\Omega},\left(c . \nabla+c_{0}\right) \varphi\right)=0$ for all $\varphi \in C_{c}^{\infty}(\Omega)$. This latter expression is well defined and states that $c_{0}=\operatorname{div} c$ in the weak sense. In particular, it implies that the distribution $\operatorname{div} c$ is in $L_{\infty, \text { loc }}(\Omega)$. In fact, we need the stronger assumption

$$
\text { (Invariance) } \quad\left(\mathbb{1}_{\Omega},\left(c . \nabla+c_{0}\right) \varphi\right)=\int_{\Omega} \mathrm{d} x\left(\left(c . \nabla+c_{0}\right) \varphi\right)(x)=0
$$

for all $\varphi \in\left(D\left(h_{N}\right) \cap L_{\infty}(\Omega)\right)_{c}$, i.e., for all bounded functions in $D\left(h_{N}\right)$ with compact support in $\bar{\Omega}$. Note that $\int_{\Omega} c . \nabla \varphi$ is well defined since $\varphi \in D\left(h_{N}\right)$ and $\int_{\Omega} c_{0} \varphi$ is well-defined since $\varphi$ has compact support and $c_{0}=\operatorname{div} c \in L_{\infty, \text { loc }}(\Omega)$.

If $\partial \Omega$ is (locally) Lipschitz then it follows from (11) and the identity $c_{0}=\operatorname{div} c$ that

$$
\int_{\Omega}\left(c . \nabla+c_{0}\right) \varphi=\int_{\Omega}(c . \nabla+\operatorname{div} c) \varphi=\int_{\Omega} \operatorname{div}(c \varphi)=\int_{\partial \Omega} \mathrm{d} S(n . c) \varphi=0
$$

for all $\varphi \in\left(D\left(h_{N}\right) \cap L_{\infty}(\Omega)\right)_{c}$ where $n$ denotes the inward normal to the boundary. Therefore, the invariance condition requires that the $c_{i}$ are degenerate on the boundary $\partial \Omega$.

LEMMA 2.5. If the growth, accretivity and invariance conditions (8), (9) and (11) are satisfied then $c . \nabla$ and $c . \nabla+c_{0}$ extend to $D\left(h_{N}\right)$ and

$$
\left(\psi,\left(c . \nabla+c_{0}\right) \varphi\right)=-(c . \nabla \psi, \varphi)
$$

for all $\psi, \varphi \in D\left(h_{N}\right)$. 
Proof. The subspace $\left(D\left(h_{N}\right) \cap L_{\infty}(\Omega)\right)_{c}$ of $D\left(h_{N}\right)$ is an algebra because $h_{N}$ is a Dirichlet form. Therefore (11), with $\varphi$ replaced by $\psi \varphi$, gives (12) for all $\psi, \varphi \in$ $\left(D\left(h_{N}\right) \cap L_{\infty}(\Omega)\right)_{c}$. In particular

$$
\left(\varphi, c_{0} \varphi\right)+2(\varphi, c . \nabla \varphi)=0
$$

for all $\varphi \in\left(D\left(h_{N}\right) \cap L_{\infty}(\Omega)\right)_{c}$. Then using the accretivity estimate (10) one has

$$
\left|\left(\varphi, c_{0} \varphi\right)\right| \leq 2\|\varphi\|_{2}\|c . \nabla \varphi\|_{2} \leq \varepsilon h_{N}(\varphi)+(\varepsilon \kappa)^{-1}\|\varphi\|_{2}
$$

for all $\varepsilon>0$ and all $\varphi \in\left(D\left(h_{N}\right) \cap L_{\infty}(\Omega)\right)_{c}$. But it follows from Lemma 2.3, using the growth condition, that $\left(D\left(h_{N}\right) \cap L_{\infty}(\Omega)\right)_{c}$ is a core of $D\left(h_{N}\right)$. Therefore, the quadratic form $\varphi \mapsto m(\varphi)=\left(\varphi, c_{0} \varphi\right)$ extends by continuity from $\left(D\left(h_{N}\right) \cap L_{\infty}(\Omega)\right)_{c}$ to $D\left(h_{N}\right)$. Hence, the corresponding bilinear form $m(\psi, \varphi)=\left(\psi, c_{0} \varphi\right)$ extends to all $\psi, \varphi \in D\left(h_{N}\right)$. But the form $l(\psi, \varphi)=(\varphi, c . \nabla \psi)$ and its adjoint $l^{*}(\psi, \varphi)=$ $l(\varphi, \psi)=(c . \nabla \varphi, \psi)$ also extend to all $\psi, \varphi \in D\left(h_{N}\right)$ by another application of (10) and the core property. Therefore (12) extends to all $\psi, \varphi \in D\left(h_{N}\right)$ and is equivalent to the statement

$$
l(\psi, \varphi)+m(\psi, \varphi)=-l^{*}(\psi, \varphi)
$$

for all $\psi, \varphi \in D\left(h_{N}\right)$.

Under the foregoing assumptions, one can now define a bilinear form $k_{N}$ with $D\left(k_{N}\right)=D\left(h_{N}\right)$ by

$$
k_{N}(\psi, \varphi)=h_{N}(\psi, \varphi)+l(\psi, \varphi)+m(\psi, \varphi)
$$

for all $\psi, \varphi \in D\left(h_{N}\right)$.

PROPOSITION 2.6. If the growth, accretivity and invariance conditions (8), (9) and (11) are satisfied then $k_{N}$ is a closed form with adjoint $k_{N}^{*}$ given by

$$
k_{N}^{*}(\psi, \varphi)=h_{N}(\psi, \varphi)-l(\psi, \varphi)
$$

for all $\psi, \varphi \in D\left(h_{N}\right)$. Moreover, if $\omega=5(2 \kappa)^{-1}$ then $k_{N}+\omega$ is accretive and continuous. In particular, the graph norms of $k_{N}+\omega$ and $h_{N}$ are equivalent and

$$
\left|k_{N}(\psi, \varphi)\right| \leq 2\left(1+\kappa^{-1}\right)\|\psi\|_{D\left(h_{N}\right)}\|\varphi\|_{D\left(h_{N}\right)}
$$

for all $\psi, \varphi \in D\left(h_{N}\right)$.

Proof. It follows from (10) and (13) extended to $\varphi \in D\left(h_{N}\right)$ that the form $\varphi \in$ $D\left(h_{N}\right) \mapsto l(\varphi)+m(\varphi)$ is bounded by $h_{N}$ with relative bound zero. Since $h_{N}$ is closed, it follows by perturbation theory that $k_{N}$ is closed.

The adjoint form is defined by $k_{N}^{*}(\psi, \varphi)=k_{N}(\varphi, \psi)$. Therefore, the adjoint relation (15) follows from Lemma 2.5. 
One next calculates with the aid of (10) and (13) that

$$
\left|k_{N}(\varphi)-h_{N}(\varphi)\right| \leq \delta h_{N}(\varphi)+\omega \delta^{-1}\|\varphi\|_{2}^{2}
$$

for each $\delta>0$ and all $\varphi \in D\left(k_{N}\right)$ with $\omega=5(2 \kappa)^{-1}$. Choosing $\delta=1$ gives $\left(k_{N}+\omega\right)(\varphi) \geq 0$ so $k_{N}+\omega$ is accretive. In addition, one has

$$
\left(k_{N}+\omega\right)(\varphi)+\|\varphi\|_{2}^{2} \leq 2 h_{N}(\varphi)+(1+2 \omega)\|\varphi\|_{2}^{2} \leq 2(1+\omega)\|\varphi\|_{D\left(h_{N}\right)}^{2}
$$

for all $\varphi \in D\left(k_{N}\right)$. Alternatively, if $\delta=1 / 2$ one obtains

$$
\begin{aligned}
& h_{N}(\varphi)+\|\varphi\|_{2}^{2} \leq 2\left(k_{N}+\omega\right)(\varphi)+(1+2 \omega)\|\varphi\|_{2}^{2} \\
& \quad \leq 2(1+\omega)\left(\left(k_{N}+\omega\right)(\varphi)+\|\varphi\|_{2}^{2}\right) .
\end{aligned}
$$

Therefore, the graph norms of $h_{N}$ and $k_{N}+\omega$ are equivalent. Hence it suffices to prove continuity with respect to the graph norm of $h_{N}$. But using the adjoint relation and the Cauchy-Schwarz inequality one finds

$$
\begin{aligned}
\left|k_{N}(\varphi, \psi)\right| & \leq\left|h_{N}(\varphi, \psi)\right|+|(c . \nabla \psi, \varphi)| \\
& \leq h_{N}(\varphi)^{1 / 2} h_{N}(\psi)^{1 / 2}+\kappa^{-1 / 2} h_{N}(\psi)^{1 / 2}\|\varphi\|_{2} \\
& \leq\left(h_{N}(\varphi)^{1 / 2}+\kappa^{-1 / 2}\|\varphi\|_{2}\right)\left(h_{N}(\psi)^{1 / 2}+\kappa^{-1 / 2}\|\psi\|_{2}\right) \\
& \leq 2\left(h_{N}(\varphi)+\kappa^{-1}\|\varphi\|_{2}^{2}\right)^{1 / 2}\left(h_{N}(\psi)+\kappa^{-1}\|\psi\|_{2}^{2}\right)^{1 / 2} \\
& \leq 2\left(1+\kappa^{-1}\right)\|\varphi\|_{D\left(h_{N}\right)}\|\psi\|_{D\left(h_{N}\right)}
\end{aligned}
$$

for all $\varphi, \psi \in D\left(h_{N}\right)$. This establishes the continuity of $k_{N}$ and the final estimate of the proposition.

Since the form $k_{N}+\omega$ is closed, accretive and continuous, it determines an operator in a canonical manner (see [21], Section 1.2.3). Therefore, one can associate an operator $K_{N}$ with the form $k_{N}$ and it follows that $K_{N}$ is an extension of the operator $K$ defined on $C_{c}^{\infty}(\Omega)$ by (1). Moreover, if $k_{D}$ denotes the restriction of $k_{N}$ to $D\left(h_{D}\right)$, the closure of $C_{c}^{\infty}(\Omega)$ with respect to $\|\cdot\|_{D\left(h_{N}\right)}$, then $k_{D}$ determines a second extension $K_{D}$ of $K$. The operators $K_{N}$ and $K_{D}$ can be interpreted as the extensions of $K$ corresponding to Neumann and Dirichlet boundary conditions, respectively. This interpretation parallels the discussion of strongly elliptic operators in Chapter 4 of [21]. The significant features of these operators are summarized in the following statement.

OBSERVATION 2.7. The operators $K_{N}$ and $K_{D}$ generate positive, strongly continuous, semigroups $S^{N}, S^{D}$ on $L_{2}(\Omega)$ satisfying bounds $\left\|S_{t}^{N}\right\|_{2 \rightarrow 2} \leq e^{\omega t},\left\|S_{t}^{D}\right\|_{2 \rightarrow 2}$ $\leq e^{\omega t}$ for all $t>0$. These semigroups extend from $L_{1}(\Omega) \cap L_{2}(\Omega)$ to strongly continuous contraction semigroups on $L_{1}(\Omega)$ and then, by interpolation, to strongly continuous semigroups on $L_{p}(\Omega)$ for all $p \in[1,2]$ satisfying the $L_{p}$-operator bounds $\left\|S_{t}^{N}\right\|_{p \rightarrow p} \leq e^{2 \omega\left(1-p^{-1}\right) t},\left\|S_{t}^{D}\right\|_{p \rightarrow p} \leq e^{2 \omega\left(1-p^{-1}\right) t}$ for all $t>0$.

The semigroup $S^{N}$ dominates $S^{D}$, i.e., $S_{t}^{N} \varphi \geq S_{t}^{D} \varphi$ for all $\varphi \geq 0$ and all $t>0$. 
The first statement follows from the standard theory of accretive operators (see, for example, [21] Chapter 1). The second statement follows because the adjoint semigroups are $L_{\infty}$-contractive by Theorem 2.15 in [21] and the ensuing discussion. Note that one uses here the relation (15) for $k_{N}^{*}$. The domination statement follows from [21], Proposition 2.23, or from the proof of Theorem 4.21.

Stannat [25] has constructed the Dirichlet extension $K_{D}$ of the non-symmetric $K$ under more general conditions. In particular, he does not require any accretivity assumption on the lower-order terms. But his arguments do not allow the construction of an analog of the Neumann extension $K_{N}$.

Next, we consider the approximation of $K_{D}$ and $K_{N}$ by a method similar to that given in Proposition 2.4 for $H_{D}$ and $H_{N}$. Let $k_{N}^{(n)}, n \in \mathbf{N}$, denote the form defined on $D\left(h_{N}\right)$ by (14) but with the coefficients $c_{i j}$ replaced by $c_{i j}^{(n)}=c_{i j}+n^{-1} \delta_{i j}$ and let $k_{D}^{(n)}$ denote the restriction of $k_{N}^{(n)}$ to $D\left(h_{D}\right)$. Further, let $K_{N}^{(n)}, K_{D}^{(n)}$ denote the associated semigroup generators. If $l_{N}$ and $l_{D}$ are again the forms associated with the Neumann and Dirichlet Laplacians on $\Omega$ then $k_{N}^{(n)}=k_{N}+n^{-1} l_{N}$ with $D\left(k_{N}^{(n)}\right)=D\left(h_{N}\right) \cap W^{1,2}(\Omega)$ and $k_{D}^{(n)}=k_{D}+n^{-1} l_{D}$ with $D\left(k_{D}^{(n)}\right)=D\left(k_{D}\right) \cap W_{0}^{1,2}(\Omega)$.

PROPOSITION 2.8. If the growth, accretivity and invariance conditions (8), (9) and (11) are satisfied then the operators $K_{D}^{(n)}$ converge to $K_{D}$ and the $K_{N}^{(n)}$ converge to $K_{N}$, both in the strong resolvent sense, as $n \rightarrow \infty$.

Proof. The proposition is a direct application of Theorem VIII.3.6 in [16]. Alternatively, it can be deduced from Theorem 5 in [20]. First consider the case of Neumann boundary conditions.

We apply Theorem VIII.3.6 of [16] by the following identification. Set $t_{n}=k_{N}^{(n)}$ and $t=k_{N}$. Then $D\left(t_{n}\right)=D\left(k_{N}\right) \cap W^{1,2}(\Omega) \subset D(t)$ and the first condition of the theorem is satisfied. Moreover, $t_{n}-t=n^{-1} l_{N}$ and since $\operatorname{Im} l_{N}=\left(l_{N}-l_{N}^{*}\right) / 2 i=0$ the second condition is also satisfied. But $t_{n}(\varphi) \rightarrow t(\varphi)$ as $n \rightarrow \infty$ for all $\varphi \in D=$ $D\left(k_{N}\right) \cap W^{1,2}(\Omega)$ and the latter set is a core of $t$ by Lemma 2.3 and Proposition 2.6. Therefore, the third condition of Theorem VIII.3.6 is satisfied. Hence $K_{N}^{(n)}$ converges in the strong resolvent sense to $K_{N}$ as $n \rightarrow \infty$.

Similar arguments apply to the forms $k_{D}^{(n)}$ and $k_{D}$ with Dirichlet boundary conditions because $D\left(h_{D}\right) \cap W_{0}^{1,2}(\Omega)$ is a core of $k_{D}$.

\section{Invariance and conservation}

The primary aim of this section is to establish the $L_{1}$-invariance of the semigroup $S^{N}$ generated by the Neumann extension $K_{N}$ of $K$. Explicitly we prove that

$$
\left(\mathbb{1}_{\Omega}, S_{t}^{N} \varphi\right)=\left(\mathbb{1}_{\Omega}, \varphi\right)
$$

for all $\varphi \in L_{1}(\Omega)$ and all $t>0$. This is clearly equivalent to the adjoint semigroup $S^{N^{*}}$ on $L_{\infty}(\Omega)$ being conservative, i.e. $S_{t}^{N^{*}} \mathbb{1}_{\Omega}=\mathbb{1}_{\Omega}$ for all $t>0$. 
The secondary aim of the section is to discuss the $L_{1}$-invariance of the semigroup $S^{D}$. It is not generally expected that $S^{D}$ satisfies the invariance property since the extension $K_{D}$ of $K$ corresponds to Dirichlet boundary conditions which are not necessarily compatible with the conservation property. We will, however, prove that $L_{1}$-invariance of $S^{D}$ is equivalent to $L_{1}$-uniqueness of $K$.

The proof of the $L_{1}$-invariance of $S^{N}$ is partially based on an argument of Auscher, Barthélemy, Bénilan and Ouhabaz [1] (see [21], Theorem 4.17) for strongly elliptic operators. The argument uses a version of Davies-Gaffney estimates [12,5] and can be modified to apply to the approximants $K_{N}^{(n)}$ used in Proposition 2.8. Consequently, one deduces that the semigroups $S^{N,(n)}$ generated by the $K_{N}^{(n)}$ are $L_{1}$-invariant. Then, one can deduce from an argument of [24], which also uses Davies-Gaffney estimates, that the $S^{N,(n)}$ are $L_{1}$-convergent to $S^{N}$. Consequently, $S^{N}$ is $L_{1}$-invariant. Both these conclusions require stronger growth assumptions than assumed in Sect. 2.

Let $\mu$ be the positive increasing function defined by (7). Then introduce the balls $B_{\mu}(r)=\{x \in \Omega: \mu(|x|)<r\}$ and let $\left|B_{\mu}(r)\right|$ denote the Lebesgue measure of the $B_{\mu}(r)$. We now introduce the strengthened growth assumption:

(Asymptotic growth II)

$$
\left\{\begin{array}{c}
\text { there are } a, b \geq 0 \text { such that } \\
\left|B_{\mu}(r)\right| \leq a e^{b r^{2}} \\
\text { for all } r \geq 1 .
\end{array}\right.
$$

If $\Omega$ is bounded and the coefficients are bounded up to the boundary then $\mu(s) \sim s$ as $s \rightarrow \infty$. Therefore, (18) is automatically satisfied. In general, this condition is a restraint on the growth of the coefficients at the boundary or at infinity. If, however, $|\Omega|=\infty$ then (18) implies the previous growth assumption (8). This follows because if (8) is false then $\mu$ is bounded and $\left|B_{\mu}(r)\right|=|\Omega|=\infty$ for all large $r$. Therefore, (18) is false.

REMARK 3.1. The analog of $v$ for the strongly elliptic operator $K_{N}^{(n)}$ with principal coefficients $C^{(n)}=C+n^{-1} I$ is given by $v_{n}=v+n^{-1}$. Therefore, the counterpart $\mu_{n}$ of $\mu$ is $\mu_{n}(s)=\int_{0}^{s}\left(n^{-1}+v\right)^{-1 / 2}$. It is evident that $\mu_{1} \leq \mu_{n} \leq \mu$. But since $v\left(0_{+}\right)>0$ it follows that $\mu \leq \lambda \mu_{1}$ with $\lambda=\left(\left(1+v\left(0_{+}\right)\right) / v\left(0_{+}\right)\right)^{1 / 2}$. Therefore, (18) implies $\lim _{s \rightarrow \infty} \mu_{n}(s)=\infty$ for all $n \geq 1$. Moreover, $B_{\mu}(r) \subseteq B_{\mu_{n}}(r) \subseteq B_{\mu_{1}}(r) \subseteq$ $B_{\mu}\left(\lambda^{-1} r\right)$ for all $n \geq 1$ and all $r>0$. Therefore (18) implies that the volume bounds $\left|B_{\mu_{n}}(r)\right| \leq a e^{b(r / \lambda)^{2}}$ are valid for all $r \geq 1$ and $n \geq 1$. Thus by increasing the value of $b$ the bounds (18) imply similar bounds for all $\mu_{n}$.

REMARK 3.2. If $v(r) \sim r^{2}$ as $r \rightarrow \infty$ then $\mu(s) \sim \log s$ and $\mu(s) \rightarrow \infty$ as $s \rightarrow$ $\infty$. Moreover, $\left|B_{\mu}(r)\right| \leq a \exp (b r)$ with $a, b>0$. Alternatively, if $v(r) \sim r^{2}(\log r)^{\alpha}$ with $\alpha \geq 0$ then $\mu(s) \sim(\log s)^{1-\alpha / 2}$ and $\mu(s) \rightarrow \infty$ as $s \rightarrow \infty$ if and only if $\alpha<2$. In this case, $\left|B_{\mu}(r)\right| \leq a \exp \left(b r^{2 /(2-\alpha)}\right)$ and the bound in (18) corresponds to $\alpha=1$.

THEOREM 3.3. Assume the accretivity, invariance and growth conditions (9), (11) and (8), (18) are satisfied. Then, the semigroup $S^{N}$ generated by the Neumann extension $K_{N}$ of $K$ satisfies the $L_{1}$-invariance condition (17). 
Proof. The first step in the proof is to modify Theorem 4.5 of [1] to deduce $L_{1}$-invariance of the semigroups $S^{N,(n)}$ generated by the strongly elliptic approximants $K_{N}^{(n)} K_{N}$ used in Proposition 2.8. Since the $K_{N}^{(n)}$ have the same lower-order terms as $K$, they satisfy the invariance condition (11). The initial problem, however, is that the result of [1] assumes the invariance property for $\varphi \in W^{1,2}(\Omega) \cap W^{1,1}(\Omega)$ while we assume invariance for $\varphi \in\left(D\left(h_{N}\right) \cap L_{\infty}(\Omega)\right)_{c}$. In fact, the proof in [1] only uses the invariance condition for $\varphi=\rho \psi$ with $\rho \in C_{c}^{\infty}(\bar{\Omega})$ and $\psi \in W^{1,2}(\Omega)$ (see [1], p. 188, or [21], p. 123). Therefore, we argue that condition (11) extends to this wider class of functions.

Fix $\rho \in C_{c}^{\infty}(\bar{\Omega})$. If $\psi \in D\left(h_{N}\right) \cap L_{\infty}(\Omega)$ then $\rho \psi \in\left(D\left(h_{N}\right) \cap L_{\infty}(\Omega)\right)_{c}$ and the invariance condition (11) gives

$$
\int_{\Omega} c_{0} \rho \psi=-\int_{\Omega}(c . \nabla)(\rho \psi) .
$$

Therefore

$$
\left|\int_{\Omega} c_{0} \rho \psi\right|=\left|\int_{\Omega}(c . \nabla)(\rho \psi)\right| \leq \kappa^{-1 / 2}\|\rho\|_{D\left(h_{N}\right)}\|\psi\|_{D\left(h_{N}\right)}
$$

by the Leibniz rule, the Cauchy-Schwarz inequality and the accretivity condition (9). Since $D\left(h_{N}\right) \cap L_{\infty}(\Omega)$ is a core of $h_{N}$, it follows that both the functions $\psi \mapsto \int_{\Omega} c_{0} \rho \psi$ and $\psi \mapsto \int_{\Omega}(c . \nabla)(\rho \psi)$ extend by continuity to functions over $D\left(h_{N}\right)$. Moreover, the extensions satisfy (19) for all $\psi \in D\left(h_{N}\right)$. Finally, suppose $\psi \in W^{1,2}(\Omega)$ and $\tilde{\rho} \in C_{c}^{\infty}(\bar{\Omega})$. Then $\tilde{\rho} \psi \in D\left(h_{N}\right)$. But one may choose $\tilde{\rho}$ such that $\rho \tilde{\rho}=\rho$. Therefore, $\rho \psi=\rho(\tilde{\rho} \psi)$ and the extension of (19) is valid for all $\psi \in W^{1,2}(\Omega)$.

The second problem with the application of the [1] theorem is that it assumes the coefficients of the elliptic operator are bounded. Therefore, we have to adapt the argument to incorporate the growth property (18).

LEMMA 3.4. There is an $\omega>0$ such that

$$
\int_{\Omega \cap B_{\mu_{n}}(R)^{\mathrm{c}}}\left|S_{t}^{N,(n)} \varphi\right|^{2} \leq e^{-(R-r)^{2}(\omega t)^{-1}} e^{\omega t}\|\varphi\|_{2}^{2}
$$

for all $\varphi \in L_{2}\left(B_{\mu}(r)\right)$ and all $t>0$ uniformly for $1<r<R$ and $n \geq 1$.

Proof. Define $\psi_{n}$ on $L_{2}(\Omega)$ as the multiplication operator $\left(\psi_{n} \varphi\right)(x)=\mu_{n}(|x|) \varphi(x)$ for $\varphi \in L_{2}(\Omega)$. Then identifying $x \in \Omega \mapsto \mu_{n}(|x|)$ as a function over $\Omega$ one has $0<\Gamma_{n}\left(\mu_{n}\right) \leq 1$ where $\Gamma_{n}$ is the carré du champ corresponding to the coefficients $C^{(n)}$. Now consider the perturbed semigroups $S_{t}^{\tau}=e^{\tau \psi_{n}} S_{t}^{N,(n)} e^{-\tau \psi_{n}}$ where $\tau \geq 0$. Then

$$
S_{t}^{N,(n)} \varphi=e^{-\tau \psi_{n}} S_{t}^{\tau} e^{\tau \psi_{n}} \varphi=e^{-\tau\left(\psi_{n}-r\right)} S_{t}^{\tau}\left(e^{\tau\left(\psi_{n}-r\right)} \varphi\right)
$$

Hence if $\varphi \in L_{2}\left(B_{\mu}(r)\right)$ then

$$
\int_{\Omega \cap B_{\mu_{n}}(R)^{\mathrm{c}}}\left|S_{t}^{N,(n)} \varphi\right|^{2} \leq e^{-2 \tau(R-r)}\left\|S_{t}^{\tau} e^{\tau\left(\psi_{n}-r\right)} \varphi\right\|_{2}^{2} \leq e^{-2 \tau(R-r)}\left\|S_{t}^{\tau}\right\|_{2 \rightarrow 2}^{2}\|\varphi\|_{2}^{2}
$$


where we have used $B_{\mu}(r) \subseteq B_{\mu_{n}}(r)$ to deduce that $\left|e^{\tau\left(\psi_{n}-r\right)} \varphi\right| \leq|\varphi|$ for $\varphi \in$ $L_{2}\left(B_{\mu}(r)\right)$. But one deduces from the strict ellipticity and uniform accretivity that there is an $\omega>0$, independent of $n$, such that

$$
\begin{aligned}
\frac{d}{d t}\left\|S_{t}^{\tau} \varphi\right\|_{2}^{2} & =-2 k_{N}^{(n)}\left(S_{t}^{\tau} \varphi\right)+2 \tau\left(\left(c . \nabla \psi_{n}\right) S_{t}^{\tau} \varphi, S_{t}^{\tau} \varphi\right)+2 \tau^{2}\left(S_{t}^{\tau} \varphi, \Gamma_{n}\left(\psi_{n}\right) S_{t}^{\tau} \varphi\right) \\
& \leq \omega\left(1+\tau^{2}\right)\left\|S_{t}^{\tau} \varphi\right\|_{2}^{2}
\end{aligned}
$$

and consequently

$$
\left\|S_{t}^{\tau}\right\|_{2 \rightarrow 2}^{2} \leq e^{\omega\left(1+\tau^{2}\right) t}
$$

for all $t>0$. Combining these estimates one obtains

$$
\int_{\Omega \cap B_{\mu_{n}}(R)^{\mathrm{c}}}\left|S_{t}^{N,(n)} \varphi\right|^{2} \leq e^{\tau^{2} \omega t-2 \tau(R-r)} e^{\omega t}\|\varphi\|
$$

for all $\tau>0$. Then setting $\tau=(R-r)(\omega t)^{-1}$ gives the statement of the lemma.

It is also necessary to have an off-diagonal estimate similar to (20) for the derivatives of the semigroup. The derivation is very similar but the estimates are $n$-dependent. First one observes that

$$
\partial_{i} S_{t}^{N,(n)} \varphi=e^{-\tau \psi_{n}} \partial_{i} S_{t}^{\tau} e^{\tau \psi_{n}} \varphi-\tau e^{-\tau \psi_{n}}\left(\partial_{i} \psi_{n}\right) S_{t}^{\tau} e^{\tau \psi_{n}} \varphi .
$$

Hence, setting $\Omega_{n}(R)^{\mathrm{c}}=\Omega \cap B_{\mu_{n}}(R)^{\mathrm{c}}$, one has

$$
\begin{aligned}
& \int_{\Omega_{n}(R)^{\mathrm{c}}} \Gamma_{n}\left(S_{t}^{N,(n)} \varphi\right) \leq 2 e^{-2 \tau(R-r)} \\
& \quad \times\left(\int_{\Omega_{n}(R)^{\mathrm{c}}} \Gamma_{n}\left(S_{t}^{\tau} e^{\tau\left(\psi_{n}-r\right)} \varphi\right)+\tau^{2} \int_{\Omega_{n}(R)^{\mathrm{c}}} \Gamma_{n}\left(\mu_{n}\right)\left|S_{t}^{\tau} e^{\tau\left(\psi_{n}-r\right)} \varphi\right|^{2}\right) .
\end{aligned}
$$

But $0 \leq \Gamma_{n}\left(\mu_{n}\right) \leq 1$. Therefore

$$
\begin{aligned}
& \int_{\Omega_{n}(R)^{\mathrm{c}}} \Gamma_{n}\left(\mu_{n}\right)\left|S_{t}^{\tau} e^{\tau\left(\psi_{n}-r\right)} \varphi\right|^{2} \\
& \quad \leq \int_{\Omega_{n}(R)^{\mathrm{c}}}\left|S_{t}^{\tau} e^{\tau\left(\psi_{n}-r\right)} \varphi\right|^{2} \leq\left\|S_{t}^{\tau}\right\|_{2 \rightarrow 2}\|\varphi\|_{2}^{2} \leq e^{\omega\left(1+\tau^{2}\right) t}\|\varphi\|_{2}^{2} .
\end{aligned}
$$

One also has

$$
\int_{\Omega_{n}(R)^{\mathrm{c}}} \Gamma_{n}\left(S_{t}^{\tau} e^{\tau\left(\psi_{n}-r\right)} \varphi\right) \leq h_{N}^{(n)}\left(S_{t}^{\tau} e^{\tau\left(\psi_{n}-r\right)} \varphi\right)
$$

Since $K_{\tau}$, the generator of $S^{\tau}$, has principal part $H_{N}^{(n)}$ one then estimates that there is an $a>0$ such that

$$
\begin{aligned}
h_{N}^{(n)}\left(S_{t}^{\tau} \chi\right) & \leq a\left(\left\|K_{\tau} S_{t}^{\tau} \chi\right\|_{2}^{2}+\left(1+\tau^{2}\right)\left\|S_{t}^{\tau} \chi\right\|_{2}^{2}\right) \\
& \leq a\left(\left\|K_{\tau} S_{t}^{\tau}\right\|_{2 \rightarrow 2}^{2}+\left(1+\tau^{2}\right)\left\|S_{t}^{\tau}\right\|_{2 \rightarrow 2}^{2}\right)\|\chi\|_{2}^{2}
\end{aligned}
$$


for all $\tau \geq 0, \chi \in L_{2}(\Omega)$ and $t>0$ uniformly in $n$. The second term is bounded as before,

$$
\left(1+\tau^{2}\right)\left\|S_{t}^{\tau}\right\|_{2 \rightarrow 2} \leq(\omega t)^{-1} e^{2 \omega\left(1+\tau^{2}\right) t},
$$

and the first term can be estimated by exploiting the holomorphy of the semigroup.

The semigroup $S^{\tau}$, viewed as acting on the complex $L_{2}$-space, is holomorphic in a sector $\Sigma\left(\theta_{n}\right)=\left\{z \in \mathbf{C}:|\arg z|<\theta_{n}\right\}$. (The sector angle is $n$-dependent since it depends on the ellipticity constant of the generator of $S^{\tau}$.) Then

$$
K_{\tau} S_{t}^{\tau} \varphi=(2 \pi i)^{-1} \int_{C_{n}} \mathrm{~d} z \frac{S_{z}^{\tau}}{(z-t)^{2}} \varphi
$$

where $C_{n}=\left\{z:\left(|z-t|<t \sin \phi_{n}\right\}\right.$ with $0<\phi_{n}<\theta_{n}$. Hence there is an $M_{n}>0$ such that

$$
\left\|K_{\tau} S_{t}^{\tau}\right\|_{2 \rightarrow 2} \leq M_{n} t^{-1} \sup _{z \in C_{n}}\left\|S_{z}^{\tau}\right\|_{2 \rightarrow 2}
$$

for all $\tau \in \mathbf{R}$ and $t>0$. Therefore, arguing as above, one obtains bounds

$$
\left\|K_{\tau} S_{t}^{\tau}\right\|_{2 \rightarrow 2} \leq M_{n}^{\prime} t^{-1} e^{\sigma_{n}\left(1+\tau^{2}\right) t}
$$

for all $t>0$ with $\sigma_{n}>0$. Combining these estimates one has the following analog of Lemma 3.4.

LEMMA 3.5. There are $M_{n}, \omega_{n}>0$ such that

$$
\int_{\Omega \cap B_{\mu_{n}}(R)^{\mathrm{c}}} \Gamma_{n}\left(S_{t}^{N,(n)} \varphi\right) \leq M_{n} t^{-1} e^{-(R-r)^{2}\left(\omega_{n} t\right)^{-1}} e^{\omega_{n} t}\|\varphi\|_{2}^{2}
$$

for all $\varphi \in L_{2}\left(B_{\mu}(r)\right)$ and all $t>0$ uniformly for $1<r<R$.

Now, we are prepared to complete the first step in the proof of Theorem 3.3, the $L_{1}$-invariance of $S^{N,(n)}$.

It clearly suffices to prove that

$$
\left(\mathbb{1}_{\Omega}, S_{t}^{N,(n)} \varphi\right)=\left(\mathbb{1}_{\Omega}, S_{S}^{N,(n)} \varphi\right)
$$

for all $s, t \in\langle 0,1\rangle$ and all $\varphi$ in an $L_{1}$-dense subspace. Therefore, we consider the subspace of $L_{2}(\Omega)$ spanned by the functions with support in a ball $B_{\mu}(r)$. Since this space contains $C_{c}^{\infty}(\Omega)$, it is certainly $L_{1}$-dense.

Fix $\varphi \in L_{2}\left(B_{\mu}(r)\right)$. Let $\eta_{m}$ denote the sequence of functions given by $\eta_{m}(x)=$ $\chi\left(m^{-1} \mu_{n}(|x|)\right)$ where $\chi$ is the function used in the proof of Lemma 2.3. It then follows that

$$
\begin{aligned}
\left(\mathbb{1}_{\Omega}, S_{t}^{N,(n)} \varphi\right)-\left(\mathbb{1}_{\Omega}, S_{s}^{N,(n)} \varphi\right) & =\lim _{m \rightarrow \infty}\left(\left(\eta_{m}, S_{t}^{N,(n)} \varphi\right)-\left(\eta_{m}, S_{s}^{N,(n)} \varphi\right)\right) \\
& =-\lim _{m \rightarrow \infty} \int_{s}^{t} d u k_{N}^{(n)}\left(S_{u}^{N,(n)} \varphi, \eta_{m}\right) .
\end{aligned}
$$


Set $\varphi_{u}=S_{u}^{N,(n)} \varphi$. Then $\varphi_{u} \in D\left(h_{N}\right)$ and

$$
\left(\mathbb{1}_{\Omega},\left(c . \nabla+c_{0}\right) \eta_{m} \varphi_{u}\right)=0
$$

by the extension of the invariance condition established at the beginning of the proof of the theorem. Therefore,

$$
k_{N}^{(n)}\left(\varphi_{u}, \eta_{m}\right)=h_{N}^{(n)}\left(\varphi_{u}, \eta_{m}\right)-\left(\left(c . \nabla \eta_{m}\right), \varphi_{u}\right) .
$$

Now $\Gamma_{n}\left(\eta_{m}\right) \leq 4 m^{-2}$, supp $\eta_{m} \subseteq B_{\mu_{n}}(2 m)$ and $\chi(s)=1$ if $s \in\langle 0,1\rangle$. Therefore,

$$
\begin{aligned}
\left|h_{N}^{(n)}\left(\eta_{m}, \varphi_{u}\right)\right| & \leq \int_{\Omega}\left|\Gamma_{n}\left(\eta_{m}, \varphi_{u}\right)\right| \\
& \leq 2 m^{-1}\left|B_{\mu_{n}}(2 m)\right|^{1 / 2}\left(\int_{\Omega \cap B_{\mu_{n}}(m)^{\mathrm{c}}} \Gamma_{n}\left(\varphi_{u}\right)\right)^{1 / 2}
\end{aligned}
$$

uniformly for $u \in\langle s, t\rangle$. Moreover, it follows from the accretivity condition (9) that one also has $\left|c . \nabla \eta_{m}\right|^{2} \leq \kappa^{-1} \Gamma_{n}\left(\eta_{m}\right)$. Therefore,

$$
\left|\left(\left(c . \nabla \eta_{m}\right), \varphi_{u}\right)\right| \leq \kappa^{-1 / 2} 2 m^{-1}\left|B_{\mu_{n}}(2 m)\right|^{1 / 2}\left(\int_{\Omega \cap B_{\mu_{n}}(m)^{\mathrm{c}}}\left|\varphi_{u}\right|^{2}\right)^{1 / 2}
$$

uniformly for $u \in\langle s, t\rangle$.

But it follows from the asymptotic growth property (18) and Remark 3.1 that there are $a, b>0$ such that $\left|B_{\mu_{n}}(2 m)\right| \leq a e^{4 b m^{2}}$ for all $m \geq 1$ uniformly in $n$. Moreover the integrals in the estimates (25) and (26) can be bounded by Lemmas 3.4 and 3.5. One then concludes that there are $M_{n}, \sigma_{n}>0$ such that

$$
\left|k_{N}^{(n)}\left(\varphi_{u}, \eta_{m}\right)\right| \leq M_{n} m^{-1} e^{2 b m^{2}} e^{-m^{2}\left(\sigma_{n} t\right)^{-1}} e^{\left(\sigma_{n} s\right)^{-1}}\left\|\varphi_{u}\right\|_{2}
$$

uniformly for $u \in\langle s, t\rangle$. Therefore if $t_{n}=\left(2 \sigma_{n} b\right)^{-1}$ then

$$
\lim _{m \rightarrow \infty}\left|k_{N}^{(n)}\left(\varphi_{u}, \eta_{m}\right)\right|=0
$$

for all $u \in\left\langle s, t_{n}\right]$. Hence (22) follows from (23) for all $s, t \in\left\langle 0, t_{n}\right]$. Then it follows for all $s, t>0$ by density, continuity, and the semigroup property.

At this stage, we have established that the semigroups $S^{N,(n)}$ are $L_{1}$-invariant. Moreover, they are $L_{2}$-convergent, as $n \rightarrow \infty$, to the semigroup $S^{N}$ as a direct corollary of Proposition 2.8. But this is not sufficient to deduce that $S^{N}$ is $L_{1}$-invariant. This requires $L_{1}$-convergence of the $S_{t}^{N,(n)}$. The $L_{2}$-convergence can, however, be converted into $L_{1}$-convergence by an argument used in [24].

It suffices to prove convergence on functions in $L_{1}(\Omega) \cap L_{2}(\Omega)$ with compact support. Assume $\varphi \in L_{1}\left(B_{\mu}(r)\right) \cap L_{2}\left(B_{\mu}(r)\right)$. Then

$$
\begin{aligned}
& \left\|\left(S_{t}^{N,(n)}-S_{t}^{N,(m)}\right) \varphi\right\|_{1} \leq\left\|\mathbb{1}_{B_{\mu}(R)}\left(S_{t}^{N,(n)}-S_{t}^{N,(m)}\right) \varphi\right\|_{1} \\
& \quad+\int_{\Omega \cap B_{\mu}(R)^{\mathrm{c}}}\left|S_{t}^{N,(n)} \varphi\right|+\int_{\Omega \cap B_{\mu}(R)^{\mathrm{c}}}\left|S_{t}^{N,(m)} \varphi\right| \\
& \leq\left|B_{\mu}(R)\right|^{1 / 2}\left\|\left(S_{t}^{N,(n)}-S_{t}^{N,(m)}\right) \varphi\right\|_{2}+2 \sup _{n \geq 1} \int_{\Omega \cap B_{\mu}(R)^{\mathrm{c}}}\left|S_{t}^{N,(n)} \varphi\right|
\end{aligned}
$$


for all $R>r$. Since $S_{t}^{N,(n)}$ is $L_{2}$-convergent to $S_{t}^{N}$ for all $t>0$, it now suffices for the $L_{1}$-convergence to prove that the last term converges to zero as $R \rightarrow \infty$ for each $t$ in a finite interval $\left\langle 0, t_{0}\right]$. But this can be established with the estimates of Lemma 3.4. It is crucial that these estimates are uniform in $n$.

It follows from Remark 3.1 that $B_{\mu}(r) \subseteq B_{\mu_{n}}(r) \subseteq B_{\mu}\left(\lambda^{-1} r\right)$ for all $n \geq 1$ and all $r>0$. Therefore, it suffices to prove that

$$
\lim _{R \rightarrow \infty} \sup _{n \geq 1} \int_{\Omega \cap B_{\mu_{n}}(R)^{\mathrm{c}}}\left|S_{t}^{N,(n)} \varphi\right|=0
$$

for all $t \in\left\langle 0, t_{0}\right]$. Now let $A_{m}=\left\{x \in \Omega: m \leq \mu_{n}(|x|)<m+1\right\}$ with $m>r$. Then there is an $\omega>0$ such that

$$
\begin{aligned}
\int_{A_{m}}\left|S_{t}^{N,(n)} \varphi\right| \leq\left(\mathbb{1}_{A_{m}}, S_{t}^{N,(n)}|\varphi|\right) & \leq\left|B_{\mu_{n}}(m+1)\right|^{1 / 2}\left(\int_{\Omega \cap B_{\mu_{n}}(m)^{\mathrm{c}}}\left|S_{t}^{N,(n)} \varphi\right|^{2}\right)^{1 / 2} \\
& \leq\left|B_{\mu_{n}}(m+1)\right|^{1 / 2} e^{-(m-r)^{2}(\omega t)^{-1}} e^{\omega t}\|\varphi\|_{2}
\end{aligned}
$$

for all $t>0$ uniformly for $n \geq 1$ by Lemma 3.4. But it follows from the growth assumption (18) and Remark 3.1 that there are $a, b>0$ such that $\left|B_{\mu_{n}}(m+1)\right|^{1 / 2} \leq a e^{b m^{2}}$ for all $m \geq 0$ uniformly for $n \geq 1$. Thus

$$
\int_{A_{m}}\left|S_{t}^{N,(n)} \varphi\right| \leq a e^{b m^{2}} e^{-(m-r)^{2}(\omega t)^{-1}} e^{\omega t}\|\varphi\|_{2}
$$

for all $t>0$ uniformly for $n \geq 1$. Therefore, one may choose $t_{0}>0$ and $a^{\prime}, \omega^{\prime}>0$ such that

$$
\int_{A_{m}}\left|S_{t}^{N,(n)} \varphi\right| \leq a^{\prime} e^{-b m^{2}} e^{\omega^{\prime}\left(t+t^{-1}\right)}\|\varphi\|_{2}
$$

for all $t \in\left\langle 0, t_{0}\right]$ uniformly for $n \geq 1$.

Finally, since we may assume $R$ is integer, it follows that

$$
\int_{\Omega \cap B_{\mu_{n}}(R)^{\mathrm{c}}}\left|S_{t}^{N,(n)} \varphi\right| \leq \sum_{m=R}^{\infty} \int_{A_{m}}\left|S_{t}^{N,(n)} \varphi\right| \leq a^{\prime}\left(\sum_{m=R}^{\infty} e^{-b m^{2}}\right) e^{\omega^{\prime}\left(t+t^{-1}\right)}\|\varphi\|_{2}
$$

for all $t \in\left\langle 0, t_{0}\right]$ uniformly for $n \geq 1$ and (27) follows immediately.

This completes the proof of Theorem 3.3.

Next, we consider the $L_{1}$-invariance property of the submarkovian semigroup $S^{D}$. The next theorem shows the invariance is equivalent to $L_{1}$-uniqueness of $H$. The theorem is a version of a result valid for divergence form operators on manifolds $[2,4,14,15]$ adapted to the current setting. It only requires the milder growth condition (8) used in Sect. 2 to derive the general structural properties of $S^{D}$.

THEOREM 3.6. Assume the growth, accretivity and invariance conditions (8), (9) and (11) are satisfied. Let $S^{D}$ denote the semigroup generated by the extension $K_{D}$ of $K$ with Dirichlet boundary conditions.

Then, the following conditions are equivalent: 
I. $K$ is $L_{1}$-unique,

II. $\left(\mathbb{1}_{\Omega}, S_{t}^{D} \varphi\right)=\left(\mathbb{1}_{\Omega}, \varphi\right)$ for all $\varphi \in L_{1}(\Omega)$ and all $t>0$.

Proof. I $\Rightarrow$ II The operator $K$ is $L_{1}$-unique if and only if $K_{D}$ is equal to the $L_{1}$-closure $\bar{K}^{1}$ of $K$. (If $K_{D}=\bar{K}^{1}$ then $K$ is $L_{1}$-unique by the Hille-Yosida theorem. Conversely, if $K_{D}$ is a strict extension of $\bar{K}^{1}$ then $K$ has an infinite number of generator extensions by a result of Arendt, Theorem 1.33 in Section A-II of [19].) But $\left(\mathbb{1}_{\Omega}, K \varphi\right)=0$ for all $\varphi \in C_{c}^{\infty}(\Omega)$ by the invariance assumption (11). Hence $\left(\mathbb{1}_{\Omega}, \bar{K}^{1} \varphi\right)=0$ for all $\varphi \in D\left(\bar{K}^{1}\right)$ or, equivalently, $\left(\mathbb{1}_{\Omega}, K_{D} \varphi\right)=0$ for all $\varphi \in D\left(K_{D}\right)$. Then, since $S_{t}^{D} D\left(K_{D}\right) \subseteq D\left(K_{D}\right)$, one has

$$
\frac{d}{d t}\left(\mathbb{1}_{\Omega}, S_{t}^{D} \varphi\right)=-\left(\mathbb{1}_{\Omega}, K_{D} S_{t}^{D} \varphi\right)=0
$$

for all $\varphi \in D\left(K_{D}\right)$ and $t>0$. Therefore $\left(\mathbb{1}_{\Omega}, S_{t}^{D} \varphi\right)=\left(\mathbb{1}_{\Omega}, \varphi\right)$ for all $\varphi \in D\left(K_{D}\right)$ and then by density for all $\varphi \in L_{1}(\Omega)$.

$\mathrm{II} \Rightarrow \mathrm{I}$ The argument follows the reasoning in Section 2 of [4] for symmetric operators. Explicitly we argue that if Condition I is false then Condition II is false.

If $K$ is not $L_{1}$-unique, then the range of $\lambda I+K$ is not dense in $L_{1}(\Omega)$ for all large positive $\lambda$. Choose $\lambda>0$ and $\varphi \in L_{\infty}(\Omega)$ with $\|\varphi\|_{\infty}=1$ such that

$$
(\varphi,(\lambda I+K) \chi)=0
$$

for all $\chi \in C_{c}^{\infty}(\Omega)$. The first key step in the proof is to establish that $\varphi \in W_{\text {loc }}^{1,2}(\Omega)$. This is a corollary of the following lemma.

LEMMA 3.7. Let $U$ be a relatively compact open subset of $\Omega$ and let $K_{U}$ denote the restriction of $K$ to $C_{c}^{\infty}(U)$.

If $K_{U}^{*}$ denotes the $L_{2}(U)$-adjoint of $K_{U}$ then $D\left(K_{U}^{*}\right) \subseteq W_{\mathrm{loc}}^{1,2}(U)$.

Proof. A similar statement for the principal part $H$ of $K$ was established in [23], Lemma 2.2, and the proof follows the reasoning of this reference.

First, $K_{U}$ is a strongly elliptic operator with bounded coefficients on $L_{2}(U)$. Strong ellipticity follows directly from the strict ellipticity assumption.

Secondly, one can extend $K_{U}$ to a strongly elliptic operator $\widehat{K}$ on $L_{2}\left(\mathbf{R}^{d}\right)$ with coefficients $\hat{c}_{i j} \in W^{1, \infty}\left(\mathbf{R}^{d}\right)$ and $\hat{c}_{i}, \hat{c}_{0} \in L_{\infty}\left(\mathbf{R}^{d}\right)$ as follows. If $C(x) \geq \mu I>0$ for all $x \in U$ one may choose a relatively compact open subset $U^{\prime}$ of $\Omega$ such that $U \subset U^{\prime}$ and $C(x) \geq(\mu / 2) I>0$ for all $x \in U^{\prime}$. Then if $\chi \in C^{\infty}\left(\mathbf{R}^{d}\right)$ with $0 \leq \chi \leq 1, \chi(x)=1$ if $x \in U$ and $\chi(x)=0$ if $x \in\left(U^{\prime}\right)^{\mathrm{c}}$ one sets $\widehat{C}=\chi C+(1-\chi)(\mu / 2) I, \hat{c}=\chi c$ and $\hat{c}_{0}=\operatorname{div}(\chi c)$. It follows that the operator $\widehat{K}$ constructed from these coefficients with domain $W^{2,2}\left(\mathbf{R}^{d}\right)$ is closed, strongly elliptic and generates a strongly continuous semigroup $\widehat{S}$ on $L_{2}\left(\mathbf{R}^{d}\right)$. Moreover, $K_{U}=\left.\widehat{K}\right|_{C_{c}^{\infty}(U)}$. This result was established for the strongly elliptic extension $\widehat{H}$ of the symmetric principal part $H$ of $K$ in the appendix of [23] and the proof for $\widehat{K}$ is very similar. Alternatively, it can be derived from the result for $H$ by perturbation theory. It also follows that the $L_{2}$-adjoint $\widehat{K}^{*}$ of 
$\widehat{K}$ is the closed strongly elliptic operator with coefficients $\hat{c}_{i j},-\hat{c}_{i}$. The adjoint $\widehat{K}^{*}$ generates the adjoint semigroup $\widehat{S}^{*}$.

Thirdly, the resolvents $(\kappa I+\widehat{K})^{-1}$ and $\left(\kappa I+\widehat{K}^{*}\right)^{-1}$ map $L_{2}\left(\mathbf{R}^{d}\right)$ to $W^{2,2}\left(\mathbf{R}^{d}\right)$ for all large $\kappa>0$. Therefore, by duality, they map $W^{-2,2}\left(\mathbf{R}^{d}\right)$ to $L_{2}\left(\mathbf{R}^{d}\right)$ and, by interpolation, they also map $W^{-\delta, 2}\left(\mathbf{R}^{d}\right)$ to $W^{2-\delta}\left(\mathbf{R}^{d}\right)$ for all $\delta \in[0,2]$.

Fourthly, if $\psi \in L_{2}\left(\mathbf{R}^{d}\right)$ then there is a distribution $K^{*}(\psi) \in W^{-2,2}\left(\mathbf{R}^{d}\right)$ such that $\left(K^{*}(\psi), \varphi\right)=(\psi, \widehat{K} \varphi)$ for all $\varphi \in C_{c}^{\infty}\left(\mathbf{R}^{d}\right)$. Therefore

$$
\kappa(\psi, \varphi)+\left(K^{*}(\psi), \varphi\right)=(\psi,(\kappa I+\widehat{K}) \varphi)
$$

for all $\varphi \in C_{c}^{\infty}\left(\mathbf{R}^{d}\right)$ and then by continuity for all $\varphi \in W^{2,2}\left(\mathbf{R}^{d}\right)$. Hence, one has the $L_{2}$-identity

$$
\psi=\kappa\left(\kappa I+\widehat{K}^{*}\right)^{-1} \psi+\left(\kappa I+\widehat{K}^{*}\right)^{-1} K^{*}(\psi)
$$

for all $\psi \in L_{2}\left(\mathbf{R}^{d}\right)$. In principle, (29) is a distributional identity but since $\left(\kappa I+\widehat{K}^{*}\right)^{-1}$ maps $W^{-2,2}\left(\mathbf{R}^{d}\right)$ to $L_{2}\left(\mathbf{R}^{d}\right)$ it is an identity between $L_{2}$-functions.

Fifthly,

$$
\left(\psi, K_{U} \varphi\right)=(\psi, \widehat{K} \varphi)=\left(K^{*}(\psi), \varphi\right)
$$

for all $\varphi \in C_{c}^{\infty}(U)$. Therefore, $\psi \in D\left(K_{U}^{*}\right)$ if and only if $K^{*}(\psi) \in L_{2}(U)$. Now fix $\psi \in D\left(K_{U}^{*}\right)$. Then $\psi \in W_{\mathrm{loc}}^{1,2}(U)$ if and only if $\eta \psi \in W^{1,2}(U)$ for all $\eta \in C_{c}^{\infty}(U)$. But one has the distributional identity

$$
K^{*}(\eta \psi)=\eta K^{*}(\psi)+K^{*}(\eta) \psi-\hat{c}_{0}(\eta \psi)-2 \Gamma(\eta ; \psi)
$$

where $\Gamma(\eta ; \psi)=\sum_{i, j=1}^{d} \hat{c}_{i j}\left(\partial_{i} \eta\right)\left(\partial_{j} \psi\right)$. The first three terms in the right hand side of this identity are in $L_{2}(U)$ because $\psi, K^{*}(\psi) \in L_{2}(U)$ and $\eta, K^{*}(\eta) \in L_{\infty}(U)$. In addition, since $\hat{c}_{i j} \in W^{1,2}\left(\mathbf{R}^{d}\right)$, one has an estimate

$$
|(\Gamma(\eta ; \psi), \varphi)| \leq a\|\psi\|_{L_{2}(U)}\|\varphi\|_{W^{1,2}\left(\mathbf{R}^{d}\right)}
$$

for all $\varphi \in C_{c}^{\infty}\left(\mathbf{R}^{d}\right)$. Hence $\Gamma(\eta ; \psi) \in W^{-1,2}\left(\mathbf{R}^{d}\right)$. Therefore, $K^{*}(\eta \psi) \in$ $W^{-1,2}\left(\mathbf{R}^{d}\right)$ by (30). Then it follows from (29) that

$$
\eta \psi=\kappa\left(\kappa I+\widehat{K}^{*}\right)^{-1} \eta \psi+\left(\kappa I+\widehat{K}^{*}\right)^{-1} K^{*}(\eta \psi) .
$$

Since $\left(\kappa I+\widehat{K}^{*}\right)^{-1}$ maps $W^{-1,2}\left(\mathbf{R}^{d}\right)$ into $W^{1,2}\left(\mathbf{R}^{d}\right)$, it follows that $\eta \psi \in W^{1,2}\left(\mathbf{R}^{d}\right)$ for all $\eta \in C_{c}^{\infty}(U)$. Therefore, $\psi \in W_{\mathrm{loc}}^{1,2}(U)$ and $D\left(K_{U}^{*}\right) \subseteq W_{\mathrm{loc}}^{1,2}(U)$.

Next, note that (28) is valid for all $\chi \in C_{c}^{\infty}(U)$ and all relatively compact open subsets $U$ of $\Omega$. Therefore, the restriction of $\varphi$ to $U$ is in the domain of the $L_{2}$-adjoint $K_{U}^{*}$ of $K_{U}$ for each $U$. It then follows from Lemma 3.7 that $\varphi \in W_{\text {loc }}^{1,2}(\Omega)$.

The second key point of the proof is given by the following proposition. 
PROPOSITION 3.8. If $\eta, \xi \in L_{\infty}(\Omega)$ are non-negative, $\eta \in W_{\mathrm{loc}}^{1,2}(\Omega)$ and

$$
(\eta,(\lambda I+K) \chi) \geq \lambda(\xi, \chi)
$$

for all non-negative $\chi \in C_{c}^{\infty}(\Omega)$ then $\eta \geq \lambda\left(\lambda I+K_{D}^{*}\right)^{-1} \xi$.

Proof. First, note that if $K_{D}$ denotes the generator of the semigroup $S^{D}$ acting on $L_{1}(\Omega)$ then $K_{D}^{*}$ is the generator of the adjoint semigroup $S^{D^{*}}$ acting on $L_{\infty}(\Omega)$.

Secondly, let $\left\{U_{n}\right\}_{n \geq 1}$ be an increasing family of relatively compact open subsets of $\Omega$ with smooth boundaries $\partial U_{n}$ and such that $\Omega=\sup _{n \geq 1} U_{n}$. Then the analysis of Sect. 2 applies to $\left.K\right|_{C_{c}^{\infty}\left(U_{n}\right)}$ acting on $L_{2}\left(U_{n}\right)$. Let $K_{(n)}$ denote the analog of $K_{D}$ with $\Omega$ replaced by $U_{n}, k_{(n)}$ the corresponding form and $S^{(n)}$ the semigroup generated by $K_{(n)}$ on $L_{2}\left(U_{n}\right)$. It follows that $S^{(n)}$ extends to a positive continuous semigroup on $L_{p}\left(U_{n}\right)$ for all $p \in[1,2]$. Moreover, $S^{(n)}$ is $L_{1}$-contractive (see Observation 2.7). Next extend $S_{t}^{(n)},\left(\lambda I+K_{(n)}\right)^{-1}$, etc. to $L_{p}(\Omega)$ by setting $S_{t}^{(n)} \varphi=S_{t}^{(n)} \mathbb{1}_{U_{n}} \varphi,\left(\lambda I+K_{(n)}\right)^{-1} \varphi=\left(\lambda I+K_{(n)}\right)^{-1} \mathbb{1}_{U_{n}} \varphi$ etc. The extended $S_{t}^{(n)}$ have all the properties of a strongly continuous semigroup except $S_{t}^{(n)} \rightarrow \mathbb{1}_{U_{n}}$ as $t \rightarrow 0$. But they are positive and contractive on $L_{p}(\Omega)$. Moreover, it follows from a standard domination result (see, for example, [21] Proposition 4.23) that $S_{t}^{(n)} \varphi \leq S_{t}^{(n+1)} \varphi$ for all positive $\varphi \in L_{p}(\Omega)$ and all $t>0$.

Next, we need the following convergence result which is well known in the symmetric case. A similar $L_{1}$-result in the non-symmetric case can be found in [25].

LEMMA 3.9. The $S_{t}^{(n)}$ converge strongly on $L_{p}(\Omega)$, for $p \in[1,2]$, to $S_{t}^{D}$.

Proof. It suffices to prove convergence on $L_{1}(\Omega)$ and $L_{2}(\Omega)$ as the convergence on $L_{p}(\Omega)$ for $p \in\langle 1,2\rangle$ follows by interpolation. Moreover, it suffices to prove the convergence of $S_{t}^{(n)} \varphi$ with $\varphi \geq 0$ since the general result follows by a decomposition argument.

First consider the bounded sequence $n \in \mathbf{N} \rightarrow s_{t}^{(n)}(\psi, \varphi)=\left(\psi, S_{t}^{(n)} \varphi\right)$ with $\varphi \in L_{1}(\Omega)$ and $\psi \in L_{\infty}(\Omega)$. If $\varphi, \psi$ are positive then for each $t \geq 0$ the sequence is positive, increasing and uniformly bounded by $\|\varphi\|_{1}\|\psi\|_{\infty}$. Hence the limit $s_{t}(\psi, \varphi)=$ $\lim _{n \rightarrow \infty}\left(\psi, S_{t}^{(n)} \varphi\right)$ exists and $0 \leq s_{t}(\psi, \varphi) \leq\|\varphi\|_{1}\|\psi\|_{\infty}$. Secondly, if $\varphi$ and $\psi$ are decomposed in terms of their positive and negative parts $\varphi_{ \pm}$and $\psi_{ \pm}$, it follows that the limit exists for all $\varphi \in L_{1}(\Omega)$ and $\psi \in L_{\infty}(\Omega)$. Moreover, $\left|s_{t}(\varphi, \psi)\right| \leq 2\|\varphi\|_{1}\|\psi\|_{\infty}$. This establishes that for each $t \geq 0$ the sequence $S_{t}^{(n)}$ converges weakly on $L_{1}(\Omega)$ to a bounded linear operator $\widehat{S}_{t}$, given by $\left(\psi, \widehat{S}_{t} \varphi\right)=s_{t}(\psi, \varphi)$. The operators $\widehat{S}_{t}$ are automatically positive and $\widehat{S}_{t} \varphi \geq S_{t}^{(n)} \varphi$ for all positive $\varphi \in L_{1}(\Omega)$. A slight variation of this argument, with $\psi, \varphi \in L_{2}(\Omega)$, establishes that the $S_{t}^{(n)}$ converge weakly to $\widehat{S}_{t}$ on $L_{2}(\Omega)$.

Thirdly, to establish that the $S_{t}^{(n)}$ converge strongly to $\widehat{S}_{t}$ on $L_{1}(\Omega)$ it suffices to prove that $\left\|\left(S_{t}^{(n)}-\widehat{S}_{t}\right) \varphi\right\|_{1} \rightarrow 0$ as $n \rightarrow \infty$ for all positive $\varphi \in L_{1}(\Omega)$. But $S_{t}^{(n)} \varphi$ converges monotonically upwards to $\widehat{S}_{t} \varphi$. Therefore,

$$
\lim _{n \rightarrow \infty}\left\|\left(S_{t}^{(n)}-\widehat{S}_{t}\right) \varphi\right\|_{1}=\lim _{n \rightarrow \infty} \int_{\Omega}\left(\widehat{S}_{t} \varphi-S_{t}^{(n)} \varphi\right)=0
$$


by the monotone convergence theorem. Similarly, strong $L_{2}$-convergence follows from weak $L_{2}$-convergence because

$$
\left\|\left(\widehat{S}_{t}-S_{t}^{(n)}\right) \varphi\right\|_{2}^{2} \leq\left(\widehat{S}_{t} \varphi,\left(\widehat{S}_{t}-S_{t}^{(n)}\right) \varphi\right)
$$

for all positive $\varphi \in L_{2}(\Omega)$ since $0 \leq S_{t}^{(n)} \varphi \leq \widehat{S}_{t} \varphi$. The $\widehat{S}_{t}$ automatically inherit the bounds $\left\|\widehat{S}_{t}\right\|_{1 \rightarrow 1} \leq 1$ and $\left\|\widehat{S}_{t}\right\|_{2 \rightarrow 2} \leq e^{\omega t}$ for all $t \geq 0$.

Fourthly, the $\widehat{S_{t}}$ form a semigroup $\widehat{S}$ because

$$
\left\|\left(S_{s}^{(n)} \widehat{S}_{t}-S_{S+t}^{(n)}\right) \varphi\right\|_{1}=\left\|S_{S}^{(n)}\left(\widehat{S}_{t}-S_{t}^{(n)}\right) \varphi\right\|_{1} \leq\left\|\left(\widehat{S}_{t}-S_{t}^{(n)}\right) \varphi\right\|_{1}
$$

for all $\varphi \in L_{1}(\Omega)$ and $s, t \geq 0$. Then, in the limit $n \rightarrow \infty$, one deduces that $\widehat{S}_{S} \widehat{S}_{t}-$ $\widehat{S}_{s+t}=0$ for all $s, t \geq 0$. Hence $\widehat{S}$ is a positive $L_{1}$-continuous contraction semigroup on $L_{1}(\Omega)$ and a positive $L_{2}$-continuous semigroup. It remains to identify the generator of $\widehat{S}$.

First, if $\varphi \in C_{c}^{\infty}(\Omega)$ then

$$
t^{-1}\left(I-\widehat{S}_{t}\right) \varphi=\lim _{n \rightarrow \infty} t^{-1} \int_{0}^{t} \mathrm{~d} s S_{s}^{(n)} K_{(n)} \varphi=t^{-1} \int_{0}^{t} \mathrm{~d} s \widehat{S}_{S} K \varphi
$$

because $K_{(n)} \varphi=K \varphi$ for all $n$ sufficiently large that $\operatorname{supp} \varphi \subset U_{n}$. It follows immediately that $\widehat{K}$, the generator of $\widehat{S}$ on $L_{2}(\Omega)$, is an extension of $K$.

Secondly, since $K$ is locally strongly elliptic it follows that $D\left(k_{(n)}\right)=W_{0}^{1,2}\left(U_{n}\right)$. Moreover, if $\omega \geq 0$ is such that $k_{N}+\omega$ is accretive then $k_{(n)}+\omega$ is accretive for all $n$. In particular, $\left(\lambda I+K_{(n)}\right)^{-1} L_{2}(\Omega)=D\left(K_{(n)}\right) \subseteq W_{0}^{1,2}\left(U_{n}\right)$ for all $\lambda>\omega$. But it follows from (16) that for each $\lambda>\omega$ there is an $a>0$ such that $\left(h_{N}+1\right)(\varphi) \leq a\left(k_{N}+\lambda\right)(\varphi)$ for all $\varphi \in D\left(k_{N}\right)$. Therefore

$$
\begin{aligned}
\left\|\left(\lambda I+K_{(n)}\right)^{-1} \varphi\right\|_{D\left(h_{D}\right)}^{2} & \leq a\left(k_{N}+\lambda\right)\left(\left(\lambda I+K_{(n)}\right)^{-1} \varphi\right) \\
& =a\left(k_{(n)}+\lambda\right)\left(\left(\lambda I+K_{(n)}\right)^{-1} \varphi\right) \\
& =a\left(\left(\lambda I+K_{(n)}\right)^{-1} \varphi, \varphi\right) \leq a(\lambda-\omega)^{-1}\|\varphi\|_{2}^{2}
\end{aligned}
$$

for all $\varphi \in L_{2}(\Omega)$.

Thirdly, the semigroups $S^{(n)}$ are strongly convergent to $\widehat{S}$ on $L_{2}(\Omega)$. Therefore, the resolvents $\left(\lambda I+K_{(n)}\right)^{-1}$ are strongly convergent to $(\lambda I+\widehat{K})^{-1}$ on $L_{2}(\Omega)$. It then follows from the Banach-Alaoglu theorem that $(\lambda I+\widehat{K})^{-1} \varphi \in D\left(h_{D}\right)$ for all $\varphi \in L_{2}(\Omega)$ (see [21], Lemma 1.32 or [17], Lemma I.2.12). Thus $D(\widehat{K}) \subseteq D\left(h_{D}\right)$.

Finally, it follows by the general structure of continuous accretive operators that $K_{D}$ is the only generator extension of $K$ with domain contained in $D\left(h_{D}\right)$ (see, for example, [16] Theorem VI.2.11). Therefore, $\widehat{K}=K_{D}$ and $\widehat{S}=S^{D}$.

End of proof of Proposition 3.8 Since $\left(\lambda I+K_{(n)}\right)^{-1}$ converges strongly to $(\lambda I+$ $\left.K_{D}\right)^{-1}$ on $L_{1}(\Omega)$ it follows that $\left(\lambda I+K_{(n)}^{*}\right)^{-1}$ is weakly* convergent to $\left(\lambda I+K_{D}^{*}\right)^{-1}$ on $L_{\infty}(\Omega)$. Moreover, the convergence is pointwise monotone since

$$
\left(\lambda I+K_{\left(n_{1}\right)}^{*}\right)^{-1} \psi \leq\left(\lambda I+K_{\left(n_{2}\right)}^{*}\right)^{-1} \psi
$$


almost everywhere for each non-negative $\psi \in L_{\infty}(\Omega)$ whenever $n_{1} \leq n_{2}$ (see, for example, [21] Proposition 4.23).

Set $\eta_{n}=\lambda\left(\lambda I+K_{(n)}^{*}\right)^{-1} \xi$. Then since $\xi \geq 0$, it follows that $\eta_{n} \geq 0, \eta_{n}=0$ on $\partial U_{n}$ and $\eta_{n} \rightarrow \lambda\left(\lambda I+K_{D}^{*}\right)^{-1} \xi$ monotonically upwards as $n \rightarrow \infty$. But

$$
\left(\eta_{n},(\lambda I+K) \chi\right)=\lambda\left(\xi, \mathbb{1}_{U_{n}} \chi\right)=\lambda(\xi, \chi)
$$

for all $\chi \in C_{c}^{\infty}\left(U_{n}\right)$. Since $(\eta,(\lambda I+K) \chi) \geq \lambda(\xi, \chi)$ for all non-negative $\chi \in$ $C_{c}^{\infty}\left(U_{n}\right)$, by assumption, it follows that

$$
\left(\left(\eta-\eta_{n}\right),(\lambda I+K) \chi\right) \geq 0
$$

for all non-negative $\chi \in C_{c}^{\infty}\left(U_{n}\right)$. Since $\eta$ is non-negative and $\eta_{n}=0$ on $\partial U_{n}$, it follows that $\eta-\eta_{n} \geq 0$ on $\partial U_{n}$. But $K$ is strongly elliptic on $L_{2}\left(U_{n}\right)$ and $\eta-\eta_{n} \in$ $W^{1,2}\left(U_{n}\right)$. Therefore, $\eta \geq \eta_{n}$ almost everywhere on $U_{n}$, by the maximum principle (see [13] Theorem 8.1). Consequently, $\eta \geq \lambda\left(\lambda I+K_{D}^{*}\right)^{-1} \xi$ almost everywhere on $\Omega$.

End of proof of Theorem 3.6 Let $\eta=\mathbb{1}_{\Omega}-\varphi$ where $\varphi \in L_{\infty}(\Omega)$ satisfies (28). Then $\eta \in W_{\text {loc }}^{1,2}(\Omega)$, by Lemma 3.7, $\eta \geq 0$ and

$$
(\eta,(\lambda I+K) \chi)=\left(\mathbb{1}_{\Omega},(\lambda I+K) \chi\right)=\lambda\left(\mathbb{1}_{\Omega}, \chi\right)
$$

for all $\chi \in C_{c}(\Omega)$ by the invariance condition (11). Hence it follows from Proposition 3.8 that $\eta \geq \lambda\left(\lambda I+K_{D}^{*}\right)^{-1} \mathbb{1}_{\Omega}$ or, equivalently, $\mathbb{1}_{\Omega}-\lambda\left(\lambda I+K_{D}^{*}\right)^{-1} \mathbb{1}_{\Omega} \geq \varphi$. Then, replacing $\varphi$ by $-\varphi$ if necessary, one may find a non-negative $f \in L^{1}(\Omega)$ such that $\int_{\Omega} \varphi f>0$. Therefore

$$
\int_{\Omega}\left(\mathbb{1}_{\Omega}-\lambda\left(\lambda I+K_{D}^{*}\right)^{-1} \mathbb{1}_{\Omega}\right) f \geq \int_{\Omega} \varphi f>0
$$

Finally

$$
\lambda \int_{0}^{\infty} d t e^{-\lambda t} \int_{\Omega}\left(\mathbb{1}_{\Omega}-S_{t}^{D^{*}} \mathbb{1}_{\Omega}\right) f=\int_{\Omega}\left(\mathbb{1}_{\Omega}-\lambda\left(\lambda I+K_{D}^{*}\right)^{-1} \mathbb{1}_{\Omega}\right) f>0 .
$$

Therefore Condition II must be false.

\section{4. $L_{1}$-uniqueness}

In this section, we use the analysis of Sects. 2 and 3 to derive criteria for $L_{1}$-uniqueness and Markov uniqueness of the operators $K$ and $H$ defined on $C_{c}^{\infty}(\Omega)$ by (1) and (2). Since Theorem 3.3 plays an essential role in the subsequent discussion, it is necessary to adopt the stronger growth condition (18).

THEOREM 4.1. Assume the accretivity, invariance and growth conditions (9), (11) and (8), (18) are satisfied. 
Then the following three conditions are equivalent:

I. $K$ is $L_{1}$-unique,

II. $\quad H$ is $L_{1}$-unique,

III. $C_{c}^{\infty}(\Omega)$ is dense in $D\left(h_{N}\right)$, i.e. $D\left(h_{N}\right)=D\left(h_{D}\right)$.

Proof. It suffices to prove that $\mathrm{I} \Leftrightarrow \mathrm{III}$ since the equivalence II $\Leftrightarrow \mathrm{III}$ follows by setting $c_{i}=0=c_{0}$.

$\mathrm{I} \Rightarrow$ III We argue by negation. If Condition III is false, i.e., if $D\left(h_{N}\right) \neq D\left(h_{D}\right)$, then $D\left(k_{D}\right) \neq D\left(k_{N}\right)$ and $k_{N}$ is a proper extension of $k_{D}$. But $K_{N}$ and $K_{D}$ generate continuous semigroups $S^{N}$ and $S^{D}$ on $L_{2}(\Omega)$ which extend to contraction semigroups on $L_{1}(\Omega)$ by Observation 2.7. Since the generators of the semigroups acting on $L_{1}(\Omega)$ must be distinct $L_{1}$-extensions of $K$, it follows that $K$ is not $L_{1}$-unique.

$\mathrm{III} \Rightarrow \mathrm{I}$ If $D\left(h_{D}\right)=D\left(h_{N}\right)$ then $D\left(k_{D}\right)=D\left(k_{N}\right), k_{D}=k_{N}$ and $K_{D}=K_{N}$. But $S^{N}$ satisfies the $L_{1}$-invariance condition (17) by Theorem 3.3. Hence $S^{D}$ satisfies the $L_{1}$-invariance condition. Then $K$ is $L_{1}$-unique by Theorem 3.6.

The theorem establishes that the criteria for $L_{1}$-uniqueness of the principal part $H$ of $K$ established in $[23,24]$ are also criteria for $L_{1}$-uniqueness of $K$. The latter were, however, derived from the stronger assumption $c_{i j} \in W_{\text {loc }}^{1, \infty}(\bar{\Omega})$, which ensures the $c_{i j}$ are locally bounded up to the boundary $\partial \Omega$, and under different growth conditions. Nevertheless, the main result of [24], Theorem 1.1, is valid under the growth conditions (8) and (18).

First, recall that $W_{\text {loc }}^{1, \infty}(\bar{\Omega})$ is the space of restrictions to $\Omega$ of functions in $W_{\mathrm{loc}}^{1, \infty}\left(\mathbf{R}^{d}\right)$. Secondly, the capacity of a measurable subset $A \subseteq \bar{\Omega}$, relative to $H$, is defined by

$$
\begin{aligned}
\operatorname{cap}(A)=\inf \left\{\|\psi\|_{D\left(h_{N}\right)}\right. & : \psi \in D \text { and there exists an open set } \\
& \left.U \subset \mathbf{R}^{d} \text { such that } U \supseteq A \text { and } \psi \geq 1 \text { a. e. on } U \cap \Omega\right\} .
\end{aligned}
$$

This definition of the capacity corresponds to the capacity relative to the Dirichlet form $h_{N}$ as defined in [3] or [11].

The following proposition is a version of Theorem 1.1 of [24].

PROPOSITION 4.2. Assume the principal coefficients $c_{i j} \in W_{\mathrm{loc}}^{1, \infty}(\bar{\Omega})$ and the growth conditions (8) and (18) are satisfied. Let $T^{D}$ denote the semigroup generated by the Friedrichs extension $H_{D}$ of $H$.

Then the following conditions are equivalent:

I. $T^{D}$ is $L_{1}$-invariant,

II. $H$ is $L_{1}$-unique,

III. $H$ is Markov unique,

IV. $\operatorname{cap}(\partial \Omega)=0$.

Proof. The proof that $\mathrm{I} \Rightarrow \mathrm{II} \Rightarrow \mathrm{III} \Leftrightarrow \mathrm{IV}$ follows by the arguments on page 86 of [24]. The implications $\mathrm{I} \Rightarrow \mathrm{II} \Rightarrow \mathrm{III} \Rightarrow \mathrm{IV}$ are local results that are not sensitive to the growth of the coefficients at infinity. Moreover, the implication IV $\Rightarrow$ III only depends on the growth condition (8). 
Finally, Condition III implies that $h_{N}=h_{D}$ since $h_{N}$ and $h_{D}$ are both Dirichlet forms. Then $T_{t}^{D}=T_{t}^{N}$, the semigroup generated by $H_{N}$, and since $T^{N}$ satisfies the $L_{1}$-invariance condition (17), by Theorem 3.3, it follows that $H$ is $L_{1}$-unique, by Theorem 3.6.

REMARK 4.3. The earlier proof that $h_{D}=h_{N}$ implies $L_{1}$-uniqueness given in Theorems 1.2 and 1.3 of [23] or Theorem 1.1 of [24] involved a complicated approximation argument dependent on capacity estimates in addition to Davies-Gaffney bounds. Now, however, the implication is a direct corollary of Theorems 3.3 and 3.6. These theorems are applicable to $H$ since the accretivity and invariance conditions, which depend only on the lower-order coefficients $c_{i}, c_{0}$, are obviously satisfied. But the proofs of these latter theorems are independent of capacity estimates. Nevertheless, the capacity estimates are the most practical approach to verifying uniqueness.

Each of the characterizations of $L_{1}$-uniqueness of $H$ given by Proposition 4.2 has a direct analog for the non-symmetric operator $K$. The Markov property has to be slightly modified in the first characterization, since $K$ is not necessarily accretive.

COROLLARY 4.4. Adopt the assumptions of Theorem 4.1.

The following conditions are equivalent:

I. $K$ is $L_{1}$-unique,

II. $\quad K$ has a unique extension on $L_{2}(\Omega)$ generating a positive continuous semigroup which extends to a continuous contraction semigroup on $L_{1}(\Omega)$.

Proof. I $\Rightarrow$ II If Condition II is false then there are two distinct extensions and the corresponding semigroups extended to $L_{1}(\Omega)$ must be distinct. But the generators of these semigroups are automatically $L_{1}$-extensions of $K$. Therefore, Condition I is false.

$\mathrm{II} \Rightarrow \mathrm{I}$ If Condition II is valid one must have $K_{N}=K_{D}$. Therefore, $D\left(h_{N}\right)=D\left(h_{D}\right)$ and $K$ is $L_{1}$-unique by Theorem 4.1.

The second criterion for $L_{1}$-uniqueness of $H$ is the $L_{1}$-invariance property of $T^{D}$. As $H$ is symmetric and $H_{D}$ self-adjoint, this is equivalent to the conservation property $T_{t}^{D} \mathbb{1}_{\Omega}=\mathbb{1}_{\Omega}$ for the semigroup $T^{D}$ acting on $L_{\infty}(\Omega)$. Similar equivalences are valid for $K$ and the semigroup $S^{D}$ generated by $K_{D}$.

COROLLARY 4.5. Adopt the assumptions of Theorem 4.1.

The following conditions are equivalent:

I. $K$ is $L_{1}$-unique,

II. the semigroup $S^{D}$ is $L_{1}$-invariant,

III. the adjoint semigroup $S^{D^{*}}$ is $L_{\infty}$-conservative.

Proof. The equivalence $\mathrm{I} \Leftrightarrow \mathrm{II}$ follows since $L_{1}$-uniqueness of $K$ is equivalent to $k_{N}=$ $k_{D}$. But if $k_{N}=k_{D}$ then $K_{N}=K_{D}$ and $S^{D}$ satisfies the $L_{1}$-invariance property by Theorem 3.3. Conversely, if $S^{D}$ is $L_{1}$-invariant then $K$ is $L_{1}$-unique by Theorem 3.6. The equivalence II $\Leftrightarrow \mathrm{III}$ follows by the adjoint relation. 
Finally, one has the capacity criterion for uniqueness.

COROLLARY 4.6. Adopt the assumptions of Proposition 4.2.

The following conditions are equivalent:

I. $K$ is $L_{1}$-unique,

II. $\operatorname{cap}(\partial \Omega)=0$.

Proof. It follows from Theorem 4.1 that $L_{1}$-uniqueness of $K$ is equivalent to $L_{1}$-uniqueness of $H$ and the latter condition is equivalent to $\operatorname{cap}(\partial \Omega)=0$ by Proposition 4.2.

\section{Examples}

We conclude with two illustrations of the assumptions used in the foregoing with one-dimensional examples. The one-dimensional situation is partially misleading as many of the significant $L_{2}$-results are independent of growth properties. The first indication of this was given by an argument of Weyl (see [6], Corollary XIII.6.15) which established that the operator $H \varphi=-\left(c \varphi^{\prime}\right)^{\prime}$ with domain $C_{c}^{\infty}(\mathbf{R})$, where $c \in W_{\text {loc }}^{1, \infty}(\mathbf{R})$ and $c>0$, is essentially self-adjoint on $L_{2}(\mathbf{R})$. Subsequently, Wintner established a related result for the symmetric operator on $L_{2}(0, \infty)$ obtained by restricting $H$ to $C_{c}(0, \infty)$. In principle, this operator has equal deficiency indices $(n, n)$ with $n=0,1$ or 2 . But Wintner demonstrated that the case $n=2$ does not occur (see [6] proof of Theorem XIII.6.14). Either $n=0$ and the operator is essentially self-adjoint or $n=1$ and $H$ has a one-parameter family of self-adjoint extensions. The different self-adjoint extensions correspond to different boundary conditions at the origin but there is no boundary condition at infinity.

A full analysis of the one-dimensional case applicable to operators on all the classical function spaces was given by Feller [8-10]. Descriptions and generalizations of Feller's results have been given by many authors (see, for example, [7,18] and references therein).

The first example summarizes the uniqueness properties of $H$ on the half-line and illustrates that a restriction on the growth at infinity is essential for $L_{1}$-uniqueness.

EXAMPLE 5.1. Let $d=1$ and $\Omega=\langle 0, \infty\rangle$. Define $H$ on $C_{c}^{\infty}(0, \infty)$ by $H \varphi=$ $-\left(c \varphi^{\prime}\right)^{\prime}$ where $c \in W_{\text {loc }}^{1, \infty}(0, \infty)$ and $c>0$. Further define $\nu$ and $\rho$ on $\langle 0,1]$ by setting $v(x)=\int_{x}^{1} d s c(s)^{-1}$ and $\rho(x)=\int_{1}^{x} d s s c(s)^{-1}$. Then the following are valid.

1. $H$ is $L_{2}$-unique, i.e. essentially self-adjoint, if and only if, $v \notin L_{2}(0,1)$.

2. $H$ is Markov unique if and only if $v \notin L_{\infty}(0,1)$.

3. $H$ is $L_{1}$-unique if and only if $v \notin L_{\infty}(0,1)$ and $\rho \notin L_{\infty}(1, \infty)$.

Point 1. is proved in [22], Proposition 2.1. The proof is based on Wintner's argument mentioned previously. Point 2. follows from Feller's analysis [8], Section 23, and Wintner's result (see also [7], Theorem 2.1 and [22], Theorem 2.4). The condition $v \notin L_{\infty}(0,1)$ states that the origin is a natural boundary in Feller's terminology. Point 3 . 
is a direct consequence of Feller's results (see also [7], Theorem 2.1). The condition $\rho \notin L_{\infty}(1, \infty)$ states that infinity is a natural boundary.

As an illustration of the growth condition in the characterization of $L_{1}$-uniqueness suppose $c(x) \sim x^{2}(\log x)^{\alpha}$ as $x \rightarrow \infty$ for some $\alpha \geq 0$. Then $\rho(x) \sim(\log x)^{1-\alpha}$ and $\rho \notin L_{\infty}(1, \infty)$ if and only if $\alpha \in[0,1]$.

The second example shows that in the absence of degeneracy of the drift coefficients on the boundary the direction of the flow is important.

EXAMPLE 5.2. Let $d=1$ and $\Omega=\langle 0, \infty\rangle$. Further let $H$ denote the operator of Example 5.1 but with $c \in W^{1, \infty}(0, \infty)$. Then define $K$ by $K \varphi=H \varphi+b \varphi^{\prime}$ with $b \in L_{\infty}(0, \infty)$. If one does not assume that the coefficient $b$ satisfies the uniform accretivity condition (9) then the uniqueness properties of $K$ depend critically on the sign of $b$.

Suppose $b \leq 0$ on the interval $\langle 0,1]$. Then Feller's criterion for $L_{1}$-uniqueness of $K$ states that the origin and infinity must both be natural boundaries. But since the coefficients $b, c$ are bounded the condition at infinity is automatically satisfied. The condition at the origin then requires that $N \notin L_{\infty}(0,1)$ where

$$
N(x)=\int_{x}^{1} \mathrm{~d} s \int_{s}^{1} \mathrm{~d} t c(s)^{-1} \exp \left(-\int_{s}^{t} b c^{-1}\right)
$$

for all $x \in\langle 0,1]$. One readily estimates that

$$
v(x) \leq N(x) \leq v(x) \exp \left(\|b\|_{\infty} v(x)\right)
$$

for all $x \in\langle 0,1]$ where one again has $v(s)=\int_{s}^{1} c^{-1}$. Therefore, $N \notin L_{\infty}(0,1)$ if and only if $v \notin L_{\infty}(0,1)$. Hence $K$ is $L_{1}$-unique if and only if $H$ is $L_{1}$-unique.

Next suppose $b>0$ on $[0,1]$. Then

$$
\begin{aligned}
N(x) & =\int_{x}^{1} \mathrm{~d} t \int_{x}^{t} \mathrm{~d} s c(s)^{-1} \exp \left(-\int_{s}^{t} b c^{-1}\right) \\
& =\int_{x}^{1} \mathrm{~d} t \int_{x}^{t} \mathrm{~d} s b(s)^{-1} \frac{d}{d s} \exp \left(-\int_{s}^{t} b c^{-1}\right) \\
& \leq \omega^{-1} \int_{x}^{1} \mathrm{~d} t\left(1-\exp \left(-\int_{x}^{t} b c^{-1}\right)\right) \leq \omega^{-1}
\end{aligned}
$$

with $\omega=\inf _{x \in\langle 0,1]} b(x)>0$. Thus $N \in L_{\infty}(0,1)$ and $K$ is not $L_{1}$-unique.

In the case of negative $b$, the drift term drives the diffusion away from the boundary point $\{0\}$ and there is no effect on the uniqueness properties. But if $b$ is positive the drift is toward the boundary point and unless the strength of the drift is small compared to the diffusion term one cannot expect uniqueness. One needs to specify a boundary condition. 


\section{Acknowledgments}

The authors are indebted to Adam Sikora for numerous discussions on the subject of this article. The second named author is indebted to Michael Roeckner for an invitation to visit Bielefeld University in November 2009 during which this research was begun. The subsequent collaboration was made possible by a visit of the first named author to Macquarie University in 2011. He wishes to thank Adam Sikora and Xuan Thinh Duong for the invitation and Macquarie University and the CNRS for financial support. The article was finally completed while the second named author was visiting the Mathematics Department of Hokkaido University at the invitation of Akitaka Kishimoto.

\section{REFERENCES}

[1] Auscher, P., Barthélemy, L., Bénilan, P., and Ouhabaz, E. M., Absence de la $L^{\infty}$-contractivité pour les semi-groupes associés aux opérateurs elliptiques complexes sous forme divergence. Potential Anal. 12 (2000), 160-189.

[2] AzEncott, R., Behavior of diffusion semi-groups at infinity. Bull. Soc. Math. France 102 (1974), 193-240.

[3] Bouleau, N., and Hirsch, F., Dirichlet forms and analysis on Wiener space, vol. 14 of de Gruyter Studies in Mathematics. Walter de Gruyter \& Co., Berlin, 1991.

[4] DAVIES, E. B., $L^{1}$ properties of second order elliptic operators. Bull. London Math. Soc. 17 (1985), 417-436.

[5] DAvies, E. B., Heat kernel bounds, conservation of probability and the Feller property. J. Anal. Math. 58 (1992), 99-119. Festschrift on the occasion of the 70th birthday of Shmuel Agmon.

[6] Dunford, N., and SchwarTZ, J. T., Linear operators. Part II: Spectral theory, selfadjoint operators in Hilbert space, vol. 7 of Interscience tracts in pure and applied mathematics. Interscience Publishers, John Wiley \& Sons, New York, 1963.

[7] EBERLE, A., Uniqueness and non-uniqueness of semigroups generated by singular diffusion operators. Lect. Notes in Math. 1718. Springer-Verlag, Berlin etc., 1999.

[8] FELlER, W., The parabolic differential equations and the associated semi-groups of transformations. Ann. Math. 55 (1952), 468-519.

[9] FElleR, W., Diffusion processes in one dimension, Trans. Amer. Math. Soc. 77 (1954), 1-31.

[10] FELLER, W., Generalized second order differential operators and their lateral conditions, Illinois J. Math. 1 (1957), 459-504.

[11] Fukushima, M., Oshima, Y., and TAKeda, M., Dirichlet forms and symmetric Markov processes, vol. 19 of de Gruyter Studies in Mathematics. Walter de Gruyter \& Co., Berlin, 1994.

[12] Gaffney, M. P., The conservation property of the heat equation on Riemannian manifolds. Comm. Pure Appl. Math. 12 (1959), 1-11.

[13] GILbARG, D., and Trudinger, N.S., Elliptic partial differential equations of second order, Grundlehren der Mathematischen Wissenschaften, Vol. 224. Springer-Verlag, Berlin-New York, 1977.

[14] GRIGOR'YAN, A., Analytic and geometric background of recurrence and non-explosion of the Brownian motion on Riemannian manifolds. Bull. Amer. Math. Soc. 36 (1999), 135-249.

[15] HASMINSKII, R. Z., Ergodic properties of recurrent diffusion processes and stabilisation of the solution of the Cauchy problem for parabolic equations. Theor. Prob. and Appl. 5 (1960) 179-196.

[16] KATO, T., Perturbation theory for linear operators. Second edition, Grundlehren der mathematischen Wissenschaften 132. Springer-Verlag, Berlin etc., 1980.

[17] MA, Z. M., and RöCKNER, M., Introduction to the theory of (non symmetric) Dirichlet Forms. Universitext. Springer-Verlag, Berlin etc., 1992.

[18] Mandl, P. Analytical treatment of one-dimensional Markov processes. Academia Publishing House of the Czechoslovak Academy of Sciences, Prague, 1968 
[19] NAGEL, R., ed., One-parameter semigroups of positive operators, Lecture Notes in Mathematics 1184, Berlin etc., 1986. Springer-Verlag.

[20] Ouhabaz, E.- M., Second order elliptic operators with essential spectrum $[0, \infty)$ on $L^{p}$. Commun. Part. Diff. Eq. 20 (1995) 763-773.

[21] OuhABAZ, E.- M., Analysis of heat equations on domains, vol. 31 of London Mathematical Society Monographs Series. Princeton University Press, Princeton, NJ, 2005.

[22] Robinson, D. W., and Sikora, A., Degenerate elliptic operators in one-dimension. J. Evol. Equ. 10 (2010), 731-759.

[23] Robinson, D. W., and Sikora, A., Markov uniqueness of degenerate elliptic operators. Ann. Scuola Norm. Sup. Pisa Cl. Sci. (2011). arXiv:0912.4536.

[24] Robinson, D. W., and SiKoRA, A., $L_{1}$-uniqueness of degenerate elliptic operators. Studia Math. 203 (2011) 79-103.

[25] Stannat, W., (Non-symmetric) Dirichlet operators on $L^{1}$ : existence, uniqueness and associated Markov processes. Ann. Scuola Norm. Sup. Pisa Cl. Sci. 28 (1999), 99-140.

El Maati Ouhabaz,

Institut de Mathématiques (IMB),

CNRS UMR 5251, Univ. Bordeaux,

33405 Talence, France

E-mail: Elmaati.Ouhabaz@math.u-bordeaux1.fr

Derek W. Robinson

Mathematical Sciences Institute (CMA),

Australian National University,

Canberra, ACT 0200,

Australia

E-mail: derek.robinson@anu.edu.au 